\title{
Stabilizing all Kähler moduli in type IIB orientifolds
}

\author{
Konstantin Bobkov, ${ }^{a}$ Volker Braun, ${ }^{b}$ Piyush Kumar, ${ }^{c}$ and Stuart Raby ${ }^{a}$ \\ ${ }^{a}$ Department of Physics, The Ohio State University, \\ 191 West Woodruff Avenue, Columbus, OH 43210, U.S.A. \\ ${ }^{b}$ Dublin Institute for Advanced Studies, \\ 10 Burlington Road, Dublin, Ireland \\ ${ }^{c}$ Department of Physics, University of California, \\ Theoretical Physics Group, Lawrence Berkeley National Laboratory, \\ 1 Cyclotron Road, Berkeley, CA 94720 U.S.A. \\ E-mail: bobkov.1@osu.edu, vbraun@stp.dias.ie, \\ kpiyush@phys.columbia.edu, raby@mps.ohio-state.edu
}

AbStRACт: We describe a simple and robust mechanism that stabilizes all Kähler moduli in Type IIB orientifold compactifications. This is shown to be possible with just one non-perturbative contribution to the superpotential coming from either a D3-instanton or D7-branes wrapped on an ample divisor. This moduli-stabilization mechanism is similar to and motivated by the one used in the fluxless $G_{2}$ compactifications of $M$ theory. After explaining the general idea, explicit examples of Calabi-Yau orientifolds with one and three Kähler moduli are worked out. We find that the stabilized volumes of all two- and four-cycles as well as the volume of the Calabi-Yau manifold are controlled by a single parameter, namely, the volume of the ample divisor. This feature would dramatically constrain any realistic models of particle physics embedded into such compactifications. Broad consequences for phenomenology are discussed, in particular the dynamical solution to the strong CP-problem within the framework.

Keywords: dS vacua in string theory, Compactification and String Models, Superstrings and Heterotic Strings, Superstring Vacua

ArXiv EPRINT: 1003.1982 


\section{Contents}

1 Introduction 1

2 Stabilizing all Kähler moduli with a single non-perturbative contribution 3

2.1 Contributions to the superpotential 3

2.2 Coordinates on the Kähler moduli space 8

$\begin{array}{ll}2.3 \text { Minimizing the potential } & 10\end{array}$

3 Explicit examples $\quad 13$

$\begin{array}{ll}3.1 & \text { Simple case - a one-parameter model } \\ \end{array}$

$\begin{array}{ll}3.2 & \text { A three-parameter model } \\ & 16\end{array}$

$\begin{array}{lll}3.2 .1 & \text { The geometry } & 16\end{array}$

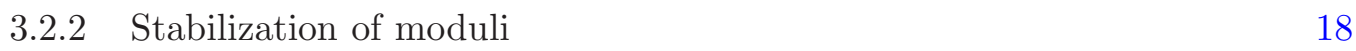

$\begin{array}{lll}\text { 3.2.3 Supersymmetry breaking and a particular choice of parameters } & 19\end{array}$

4 Consistency condition for the single condensate/instanton approximation

5 Phenomenological consequences $\quad 22$

$\begin{array}{lll}5.1 \text { A chiral spectrum } & 22\end{array}$

$\begin{array}{ll}5.2 & \text { Mediation of supersymmetry breaking }\end{array}$

5.3 Dynamical solution to the strong CP-problem 23

6 Conclusions 25

\section{Introduction}

One of the central goals of string phenomenology is to explain how particular values of low-energy physics parameters, such as the fine-structure constant or the electron Yukawa coupling, arise from a fundamental theory with no free parameters. Within the context of string theory, the values of these parameters are tied to the vacuum expectation values (vevs) of moduli. However, at a classical level, ${ }^{1}$ the moduli fields are massless and do not have fixed non-zero vevs. Therefore, in order to be able to explain the values of low-energy physics parameters and to be able to do realistic phenomenology, the moduli must be stabilized. The issue of moduli stabilization also has important implications for supersymmetry breaking and the cosmological constant.

Considerable progress has been made in the field of moduli stabilization within various corners of string theory, such as Type IIA [3, 27, 42, 70, 72], Type IIB [11, 36, 40, 41, 58,

\footnotetext{
${ }^{1}$ With no background fluxes.
} 
60, 64, 65], Heterotic $[13,29,38,55,67]$ and $G_{2}$ compactifications of $M$ theory $[2,4-6,30]$. The simplest recipe for moduli stabilization and constructing vacua with a small positive cosmological constant (de Sitter vacua) within Type IIB string theory was proposed by Kachru, Kallosh, Linde, and Trivedi [58] (KKLT). They considered a toy model with one Kähler modulus in which the dilaton and complex structure moduli were stabilized by flux contributions to the superpotential [39, 50], while the Kähler modulus $T$ was stabilized by a non-perturbative contribution to the superpotential. The non-perturbative contribution can in general arise from Euclidean D3-brane instantons or from strong gauge dynamics on stacks of D7-branes wrapping four-cycles, or divisors $D_{i}$, inside the Calabi-Yau manifold, when certain topological conditions are satisfied.

It is commonly believed, at least in the simplest setup where the Kähler potential contains no perturbative $\alpha^{\prime}$ or string loop corrections, that it is necessary for the nonperturbative part of the superpotential to contain at least $h_{+}^{11}$ linearly independent divisors $D_{i}$ in order to fix all $h_{+}^{11}$ Kähler moduli $\tau_{i}=\operatorname{Re} T_{i}$. Thus, one imagines the following superpotential:

$$
W=W_{0}+\sum_{i} A_{i} e^{-\frac{2 \pi}{c_{i}} \sum_{k} n_{k}^{(i)} T_{k}}
$$

where $W_{0}, A_{i}, c_{i}, n_{k}^{(i)}$ are constants (more on this in section 2). Following the KKLT proposal, several explicit examples of Calabi-Yau manifolds with few Kähler moduli were constructed in which the above approach to moduli fixing was successfully implemented [40, 41]. A consequence of the above mechanism is that the pseudoscalar partners of the Kähler moduli $\chi_{i}=\operatorname{Im} T_{i}$ are also generically stabilized with masses comparable to that of the $\tau_{i}$.

Although the above mechanism works for simple cases with a few Kähler moduli, one faces a number of challenges in extending it to quasi-realistic string compactifications, which could describe low-energy particle physics. First, realistic compactifications describing the many low energy parameters of particle physics are expected to contain many moduli. Thus, one generically expects the Calabi-Yau manifold $X$ to contain many Kähler moduli, with $h_{+}^{11}(X)=\mathcal{O}(100)$. Finding explicit examples with such a large number of linearly independent divisors contributing to the superpotential is a daunting task, as one must ensure that the appropriate topological condition (zero-mode structure) is satisfied for each linearly independent divisor. Second, as pointed out in [20], the Kähler modulus, which measures the volume of any four-cycle containing a chiral spectrum may not be stabilized purely by non-perturbative effects in the superpotential as in eq. (1.1). This is particularly relevant for the visible sector four-cycle as it contains a chiral spectrum. In such cases, the correct counting of zero-modes implies that exponential terms in the above superpotential appear with field-dependent prefactors containing gauge invariant combinations of chiral matter fields. Thus, in order to fix all such four-cycles, the matter fields must also be dynamically fixed in a phenomenologically viable way. However, for the visible sector such operators must have vanishing vevs for phenomenological reasons; therefore the corresponding superpotential contributions are zero. Finally, even if one comes up with a mechanism to stabilize such Kähler moduli, say by perturbative $\alpha^{\prime}$ or string-loop effects $[16,35,49]$, a detailed analysis of vacua generated by such a superpotential depends 
on a large number $\gtrsim h_{+}^{11}$ of independent parameters that enter the superpotential, making it quite intractable to make robust predictions relevant for particle phenomenology.

In this work, we advocate a different approach to fixing the Kähler moduli that is largely motivated by the general results obtained in the fluxless $G_{2}$ compactifications of Mtheory [4-6]. There, it is possible to stabilize all the moduli even when the non-perturbative superpotential receives contributions from a single associative three-cycle in a form of two gaugino condensates. Thus, here we consider Calabi-Yau orientifolds $X$ containing fewer than $h_{+}^{11}(X)$ divisors contributing to the superpotential. In the extreme case it is possible to restrict to a single divisor $D \in X$, which contributes to the non-perturbative superpotential in a form of a gaugino condensate or an instanton. The main claim of the paper is that even though the superpotential depends only on a single linear combination of Kähler moduli $\tau_{i}$, they can be stabilized self-consistently while satisfying the supergravity approximation. To be precise, we will show that this is the case if and only if the divisor $D$ is ample. Then all the four-cycle volumes $\tau_{i}$ and two-cycle volumes $t_{i}$ will be automatically stabilized inside the Kähler cone. We will define and explain this in detail in subsection 2.1.

The plan of the paper is as follows. The contributions to the superpotential and the conditions required are reviewed in subsection 2.1 within the context of the original KKLT proposal. subsection 2.2 discusses some general properties of the Kähler moduli space of Type IIB orientifolds which are crucial in performing explicit computations without specifying a particular orientifold. In subsection 2.3 , the minimization of the scalar potential and stabilization of moduli within the above framework is described in detail, where the results of subsection 2.2 are utilized. In section 3, explicit examples of Calabi-Yau orientifolds realizing the moduli stabilization procedure described in section 2 are provided. The general formalism developed in section 2 is applied to these particular cases. In section 4 , we describe conditions under which our results hold parametrically even in general cases with multiple contributions to the superpotential. Finally, section 5 is a brief discussion of the broad phenomenological consequences of this framework, followed by conclusions in section 6 .

\section{Stabilizing all Kähler moduli with a single non-perturbative contribu- tion}

\subsection{Contributions to the superpotential}

In the proposal of KKLT, the superpotential is given by a sum of the tree-level flux contributions $[37,56,71]$ that fix the dilaton and complex structure moduli $[39,50]$,

$$
W_{0}=\int_{X} G_{3} \wedge \Omega
$$

combined with non-perturbative contributions generated by strong gauge dynamics on stacks of D7-branes or Euclidean D3-brane instantons wrapping divisors $D_{i} \subset X$. Their volumes are

$$
\operatorname{Vol}\left(D_{i}\right)=\operatorname{Re}\left(\sum_{k} n_{k}^{(i)} T_{k}\right),
$$


where $n_{k}^{(i)} \in \mathbb{Z}$ specify the homology class $\left[D_{i}\right] \in H_{4}(X, \mathbb{Z})$ of the $i$-th divisor. The combined superpotential is then given by

$$
W=W_{0}+\sum_{i} A_{i} e^{-\frac{2 \pi}{c_{i}} \sum_{k} n_{k}^{(i)} T_{k}}
$$

where $c_{i}$ is the dual Coxeter number of the condensing gauge group. ${ }^{2}$ The complexstructure and dilaton moduli are stabilized at a high scale, close to the Kaluza-Klein scale. Moreover, although $W_{0}$ is generically $\mathcal{O}(1)$ in string units, it can be shown that by choosing special values of the integer-quantized fluxes it is possible to tune $W_{0}$ to a small value $[51,52]$. This turns out to be crucial, as will be explained later.

Moving on to the non-perturbative contribution, it is important to note that not every D3-instanton (or gaugino condensate on a spacetime-filling D7 brane) wrapped on a divisor $D \subset X$ contributes to the superpotential. One has to analyze the zero-modes, which is more complicated than the analogous count for M5-branes [73]. In particular, it depends not only on the arithmetic genus $\chi\left(D, \mathscr{O}_{D}\right)$ but also on the details of the orientifold action [17,64, 68]. Note that one can use the orientifold to split the arithmetic genus into

$$
\chi_{ \pm}\left(D, \mathscr{O}_{D}\right)=\sum_{n=0}^{2}(-1)^{n} h_{ \pm}^{n}\left(D, \mathscr{O}_{D}\right) .
$$

In the absence of fluxes or intersections with other D-branes, a necessary condition for a D3-instanton in an O3/O7 orientifold to contribute is $[18,19]$

$$
\chi_{+}-\chi_{-}=1
$$

Within the above setup, background fluxes are required to be present in order to stabilize the complex structure and dilaton moduli and give rise to the term $W_{0}$ in eq. (2.3). However, the above criterion still holds if the fluxes are such that they do not change the zero mode counting of the D3-instanton. For simplicity, this will be assumed from now on. Moreover, the coefficient $A$ might be zero depending on the topology of the instanton moduli space. For simplicity, we will assume that $A \neq 0$ and is an $O(1)$ complex number in the remainder of this paper.

In fact, "fractional instantons" can relax the above condition on the divisor $D$. Their microscopic origin is a stack of D7-branes, which fill spacetime in addition to wrapping the divisor, and give rise to gaugino condensation at low energies. In this case, a divisor with $\chi_{+}-\chi_{-} \geq 1$ might also contribute to the non-perturbative superpotential under some circumstances [53]. In particular, gaugino condensation can still occur in the presence of charged matter on the stack if the charged matter gets a mass at sufficiently high scales leaving a pure gauge theory at low energies. In fact, this is to be expected if the matter is vector-like. The effect on the superpotential will be an exponential term with $c_{i}>1$ in eq. (2.3). Thus if $\chi_{+}-\chi_{-}=1$ for a given divisor, there is either a D3instanton contribution (in the absence of further branes) or a gaugino condensate (if there

\footnotetext{
${ }^{2}$ For example, $c_{i}=N$ for $\mathrm{SU}(N)$ or $c_{i}=1$ for a single instanton.
} 
are stacks of D7-branes wrapping the divisor). On the other hand, for $\chi_{+}-\chi_{-}>1$ the only non-perturbative contribution could arise from a stack of D7-branes (with an appropriate spectrum) wrapping the divisor. The formal computation of the moduli vevs is the same in both cases, however, since we can effectively rescale $T_{k} \mapsto c_{i} T_{k}$ for the case of a gaugino condensate. For concreteness, therefore, we will mostly discuss D3-instantons.

A sufficient (but overly strong) condition ${ }^{3}$ for the D3-instanton to contribute to the superpotential is to demand that it is rigid and ample. Let us quickly review these notions:

- A divisor $D$ is rigid if it cannot be deformed. Thinking of the divisor as the zeroes of a section in a line bundle $\mathscr{O}(D)$, this is precisely the case if the section is unique up to an overall constant, that is, $h^{0}(X, \mathscr{O}(D))=1$. On a Calabi-Yau manifold, this is equivalent to $h^{02}(D)=0$.

- A divisor is ample 4 if and only if the Poincaré isomorphism $H_{4}(X, \mathbb{Z}) \simeq H^{2}(X, \mathbb{Z})$ identifies it with a cohomology class that can be represented by the Kähler form of a smooth Calabi-Yau metric. We will have much more to say about this condition on the next page.

For now, note that ample divisors enjoy many favorable properties. In particular, the Kodaira vanishing forces $h^{q}(X, \mathscr{O}(D))=0$ for all $q>0$, and the Lefshetz hyperplane theorem identifies $h^{p q}(X)=h^{p q}(D)$ for all $p+q<2$ as well as $\pi_{i}(X)=\pi_{i}(D)$ for $i \leq 1$.

This then leads to the following zero mode spectrum on the divisor, see eq. (3.7):

- $D$ ample $\Rightarrow h^{00}(D)=1$ and $h^{01}(D)=0$.

- $D$ rigid $\Rightarrow h^{02}(D)=0$.

In particular we see that $\chi_{+}=1, \chi_{-}=0$, and eq. (2.5) is automatically satisfied.

For simple cases of Calabi-Yau threefolds $X$ with one (or few) Kähler moduli, one may try to construct $h_{+}^{11}(X)$ rigid and linearly independent divisors. The corresponding D3-instantons would contribute $h_{+}^{11}(X)$ non-perturbative terms in the superpotential and hence stabilize all the Kähler moduli. However, as pointed out in the introduction it is

\footnotetext{
${ }^{3}$ Here and in the following we will implicitly always assume that $D$ is smooth and maps to itself under the orientifold action. Moreover, the orientifold planes are only O3/O7 (no O5/O9).

${ }^{4}$ Not to be confused with very ample. A very ample divisor is one with particularly many sections, namely, so many sections that they can be used to define a Kodaira embedding. The relation between these notions is that:

- A very ample divisor is ample.

- A sufficiently large multiple of an ample divisor is very ample.

However, note that the D7-tadpole limits the multiplicities of allowed divisors. On simple manifolds (for example, projective space) these two notions coincide. But, morally speaking, the more complicated the manifold is the more ample-but-not-very divisors are there. In section 3 we will construct some explicit examples where, in physicists terms, discrete Wilson lines are used to project out all deformations of the divisor.
} 
clear that for realistic compactifications with large $h_{+}^{11}(X)$, such a procedure is quite difficult. The intuition that one needs $h_{+}^{11}(X)$ linearly-independent divisors $D_{i}$ contributing to the superpotential in order to stabilize all Kähler moduli comes from Type IIB compactifications which admit an $F$-theory lift. Within $F$-theory compactifications on a smooth elliptically fibered Calabi-Yau fourfold with a Fano base, this is, in fact, a theorem if one only allows smooth divisors contributing to the superpotential [69].

Motivated by the general results obtained in fluxless $G_{2}$ compactifications of Mtheory [4], in this paper we show that it is possible to stabilize all $h_{+}^{11}(X)$ Kähler moduli in Type IIB orientifold compactifications containing fewer than $h_{+}^{11}(X)$ divisors contributing to the superpotential. In fact, it is possible to restrict to a single divisor $D \in X$, which contributes to the non-perturbative superpotential in the form of a single D3-instanton or gaugino condensate. We will demonstrate that, contrary to the naive expectation, even when the superpotential

$$
W\left(T_{i}\right)=W_{0}+A e^{-\frac{2 \pi}{N} \sum_{i=1}^{h_{+}^{11}} n_{i} T_{i}}
$$

depends only upon the single linear combination $\vec{n} \cdot \vec{T}$, all four-cycle volumes (Kähler moduli) $\tau_{i}$ and all two-cycle volumes $t_{i}$ can be stabilized self-consistently while satisfying the supergravity approximation. For explicitness, we will assume from now on that there is, indeed, only a single contribution to the superpotential.

The condition that all volumes of (dimension 2, 4, and 6) holomorphic submanifolds are positive can be easily formulated in terms of the Kähler form. Its cohomology class $[\omega] \in H^{1,1}(X)$ is parametrized by the Kähler moduli, but clearly not all values ${ }^{5}$ are allowed. The values that can be realized by smooth Calabi-Yau metrics form the so-called Kähler cone

$$
\left\{[\omega] \mid \omega=g_{i \bar{j}} \mathrm{~d} z^{i} \wedge \mathrm{d} z^{\bar{j}}\right\}=\mathrm{Kc}(X) \subset H^{1,1}(X) .
$$

In fact, the Kähler cone is a convex cone given by a set of linear inequalities in the 2-cycle volumes $t_{i}$. In simple cases, like the examples we will encounter later on, the Kähler cone is just the cone over a simplex. Then, perhaps after a linear change of coordinates, the Kähler cone is

$$
\operatorname{Kc}(X)=\left\{\vec{t} \mid t_{i}>0\right\} \subset H^{1,1}(X) .
$$

We can now be more explicit in the definition of an ample divisor. As we mentioned above, they are identified with potential Kähler forms. Explicitly, a divisor defines a line bundle whose first Chern class is the associated cohomology class. Therefore, a divisor $D$ is ample if and only if $c_{1}(\mathscr{O}(D)) \in \mathrm{Kc}(X)$. If we take the basis $\left[D_{i}\right]$ of $H_{4}(X, \mathbb{Z})$ to be Poincaré dual to the two-cycle classes used in eq. (2.8), then

$$
D=\sum_{i=1}^{h_{+}^{11}} n_{i} D_{i} \quad \text { is ample } \quad \Leftrightarrow \quad n_{i}>0, \quad i=1, \ldots, h_{+}^{11} .
$$

Note that, at this point, we have two seemingly independent elements of the Kähler cone:

- The (smooth) Calabi-Yau metric defines a class $[\omega] \in \mathrm{Kc}(X) \subset H^{1,1}(X)$.

\footnotetext{
${ }^{5}$ For example, if $[\omega]$ is a Kähler class then - $[\omega]$ cannot be the cohomology class of a Kähler form.
} 
- The ample divisor $D$ wrapped by the D3-instanton defines a class $c_{1}(\mathscr{O}(D)) \in$ $\operatorname{Kc}(X) \subset H^{1,1}(X)$.

The point of this paper is to show that the supergravity action, with the superpotential term generated by $D$, dynamically adjusts the Kähler moduli (that is, the coordinates of $[\omega])$ such that these are the same up to an overall rescaling of the volume. That is, the superpotential contribution of a single divisor stabilizes all Kähler moduli at a specific point

$$
[\omega]=\lambda \cdot c_{1}(\mathscr{O}(D)), \quad 0<\lambda \in \mathbb{R} .
$$

Therefore, the Calabi-Yau metric is smooth (and all volumes are positive) if and only if $D$ is ample.

Some important comments are warranted. Within the context of the theorem in $F$ theory mentioned above this implies that these Type IIB compactifications do not admit a simple lift to $F$-theory with the stated properties. In particular, the arithmetic genus $\chi\left(D, \mathscr{O}_{D}\right)<0$ for a smooth ample divisor in a Calabi-Yau fourfold [69]. This implies that the $F$-theory lift of these single-instanton compactifications, if possible, must employ non-smooth divisors in the corresponding Calabi-Yau fourfold. However, within Type IIB, there is no such problem. The direction of the inequality depends in an essential way on the dimension and, in fact, $\chi\left(D, \mathscr{O}_{D}\right)>0$ for an ample divisor in a Calabi-Yau threefold. In particular, condition eq. (2.5) can be already satisfied with smooth ample divisors.

We will thus consider a simple class of compactifications where the superpotential is given by eq. (2.6) above and the Kähler potential ${ }^{6}$ is given by

$$
K\left(T_{i}, \bar{T}_{i}\right)=-2 \ln V_{X}
$$

where the six-dimensional volume $V_{X}\left(\tau_{i}\right)$ is a homogeneous function of degree $\frac{3}{2}$. Note that, while parameter $A$ in eq. (2.6) is independent of the Kähler moduli, it does depend on the complex structure moduli and the D3-brane positions if D3-branes are present. Since the complex structure moduli are frozen by the fluxes near the Kaluza-Klein (KK) scale, their dynamics is essentially decoupled and one can treat $A$ as a constant parameter, which can be discretely adjusted by scanning over the fluxes, just like $W_{0}$. To further simplify the analysis, we will consider a setup with no D3-branes. In addition, in the general case additional non-perturbative terms are present in the superpotential, such as from multiply wrapped D3-instantons. Thus, strictly speaking the single term in eq. (2.6) should be regarded as the dominant contribution in a series of contributions that determines the moduli vevs, while the remaining terms are subleading and do not affect the moduli vevs. In section 4 , we demonstrate that such a truncation can be made parametrically self-consistent, implying that the results obtained are quite robust.

As will be shown in subsection 2.3, with the superpotential in eq. (2.6) and the Kähler potential in eq. (2.11), it turns out that the supergravity scalar potential has a supersym-

\footnotetext{
${ }^{6}$ Here we will ignore the complex structure and dilaton contributions $\mathcal{K}$ to the Kähler potential. Including such terms simply rescales the parameters of the superpotential by an overall multiplicative factor.
} 
metric Anti-de Sitter (AdS) extremum, the same as in the KKLT proposal. ${ }^{7}$ In order to obtain de Sitter vacua, therefore, additional positive contributions to the scalar potential are required. This can be achieved either by introducing matter fields in the superpotential and Kähler potential and including their dynamics via $F$ and $D$-terms $[8,9,34,62]$, or by including explicit supersymmetry violating terms in the potential, such as due to adding a small number of anti D3-branes, which are trapped at the bottom of the warped throat [61] generated in these flux compactifications. The latter route was taken by KKLT [58], and for simplicity we will follow the same approach. The potential is then given by

$$
V=e^{K}\left(G^{i \bar{j}} D_{i} W \overline{D_{j} W}-3|W|^{2}\right)+\frac{D}{V_{X}^{r}} .
$$

where $r=2$ when warping effects are negligible and $r=4 / 3$ when warping is strong, ${ }^{8}$ while $D$ is a constant that depends on the warp factor. In a companion paper [1], we study alternative ways of obtaining de Sitter vacua.

\subsection{Coordinates on the Kähler moduli space}

In this section we discuss some properties of the Kähler moduli space metric in Type IIB Calabi-Yau orientifolds. These properties are completely general and are true for all Calabi-Yau threefolds. Therefore, they allow us to perform explicit computations without specifying a particular manifold.

In mathematics, the Kähler moduli space is usually parametrized by volumes $t_{i}$ of curves (two-cycles). Here, $i \in\left\{1, \ldots, h_{+}^{11}\right\}$ runs over a basis of orientifold-invariant curves. Using these coordinates, the volume is a cubic polynomial

$$
V_{X}\left(t_{i}\right)=\sum d_{i j k} t_{i} t_{j} t_{k}
$$

where $d_{i j k}$ is the cup product ${ }^{9}$ of the basis $(1,1)$-forms. However, physicists prefer different coordinates. They parametrize the complexified Kähler moduli space $\mathcal{M}_{K}$ of the orientifold $X$ with holomorphic coordinates $T_{i}$ given by

$$
T_{i}=\tau_{i}+i \chi_{i}, \quad 1 \leq i \leq h_{+}^{11}(X),
$$

where $\tau_{i}$ are the volumes of four-cycles inside the manifold $X$ and $\chi_{i}$ are the axions corresponding to the periods of the Ramond-Ramond four-form. As volumes of four-cycles, the $\left\{\tau_{i}\right\}$ variables are quadratic polynomials in the $\left\{t_{i}\right\}$. However, despite the fact that we use the index " $i$ " in both cases, the basis of four-cycles is independent of the basis of two-cycles. A particularly nice relative basis choice is where the cycles are Poincaré dual, which amounts to setting

$$
\tau_{i}=\frac{\partial V_{X}}{\partial t_{i}}
$$

\footnotetext{
${ }^{7}$ There may also exist multiple Anti-de Sitter vacua with spontaneously broken supersymmetry similar to those found in [6]. However, we restrict our discussion to the supersymmetric extremum because it is directly related to the de Sitter minimum obtained after the uplifting.

${ }^{8}$ We would like to thank Kiwoon Choi and Hans Peter Nilles for reminding this important detail to us.

${ }^{9}$ Using the Poincaré isomorphism $H^{2}(X, \mathbb{Z})=H_{4}(X, \mathbb{Z})$, the $d_{i j k}$ are the triple intersection number of the corresponding divisors.
} 
We will always use this basis choice in the following. In order to transform the two coordinates into each other we need the Jacobian matrix, which is symmetric because

$$
\frac{\partial \tau_{i}}{\partial t_{j}}=\frac{\partial^{2} V_{X}}{\partial t_{i} \partial t_{j}}=\frac{\partial \tau_{j}}{\partial t_{i}}
$$

Therefore,

$$
\frac{\partial V_{X}}{\partial \tau_{i}}=\sum_{j=1}^{h_{+}^{11}} \frac{\partial V_{X}}{\partial t_{j}} \frac{\partial t_{j}}{\partial \tau_{i}}=\sum_{j=1}^{h_{+}^{11}} \tau_{j} \frac{\partial t_{j}}{\partial \tau_{i}}=\sum_{j=1}^{h_{+}^{11}} \tau_{j} \frac{\partial t_{i}}{\partial \tau_{j}}=\frac{1}{2} t_{i},
$$

where we used Euler's homogeneous function theorem for $t_{i}$ being a homogeneous function of the $\tau_{j}$ of degree $\frac{1}{2}$. The homogeneity of $V_{X}$ and $\tau_{i}$ is the key property that will be used in what follows.

The classical moduli space metric (not including possible quantum corrections) is determined by the Kähler potential

$$
K=-2 \ln V_{X}
$$

Note that the dimensionless volume $V_{X}=\operatorname{Vol}(X) / \ell_{s}^{6}$ is a homogeneous function of degree $\frac{3}{2}$ in the $\tau_{i}$, where $\ell_{s}$ is the string scale. Let us define the following derivatives with respect to the four-cycles ${ }^{10}$

$$
K_{i}=\frac{\partial K}{\partial \tau_{i}}=2 \frac{\partial K}{\partial T_{i}}=-\frac{2}{V_{X}} \frac{\partial V_{X}}{\partial \tau_{i}}=-\frac{t_{i}}{V_{X}}, \quad K_{i j}=\frac{\partial^{2} K}{\partial \tau_{i} \partial \tau_{j}}=4 \frac{\partial^{2} K}{\partial T_{i} \partial \bar{T}_{\bar{j}}}=4 G_{i \bar{j}},
$$

where $G_{i \bar{j}}$ is the Kähler metric controlling the kinetic terms in the Lagrangian. Since $V_{X}$ is a homogeneous function of degree $\frac{3}{2}$, the first derivatives satisfy Euler's homogeneous function theorem

$$
3 V_{X}=\sum_{i=1}^{h_{+}^{11}} \frac{\partial V_{X}}{\partial t_{i}} t_{i}=\sum_{i=1}^{h_{+}^{11}} \tau_{i} t_{i} \quad \Leftrightarrow \quad \sum_{i=1}^{h_{+}^{11}} \tau_{i} K_{i}=-3 .
$$

This suggests to introduce new variables $a_{i}$ defined by

$$
a_{i}=-\frac{1}{2} \tau_{i} K_{i}=\frac{\tau_{i} t_{i}}{2 V_{X}}
$$

with no sum over $i$ implied on the right hand side. Clearly, the $a_{i}$ are of homogeneous degree 0 in the $\tau_{i}$ (and the $t_{i}$ ), decoupling the overall volume. In particular, we see from eq. (2.21) that the $a_{i}$ satisfy

$$
\sum_{i=1}^{h_{+}^{11}} a_{i}=\frac{3}{2} .
$$

To summarize, there are three coordinate systems on the Kähler moduli space:

\footnotetext{
${ }^{10}$ For reference we note that, using eq. (2.14), the derivatives are related by

$\frac{\partial}{\partial \tau_{i}}=\frac{\partial}{\partial T_{i}}+\frac{\partial}{\partial \bar{T}_{i}}, \quad \frac{\partial}{\partial \chi_{i}}=i \frac{\partial}{\partial T_{i}}-i \frac{\partial}{\partial \bar{T}_{i}}, \quad \frac{\partial}{\partial T_{i}}=\frac{1}{2} \frac{\partial}{\partial \tau_{i}}-\frac{i}{2} \frac{\partial}{\partial \chi_{i}}, \quad \frac{\partial}{\partial \bar{T}_{i}}=\frac{1}{2} \frac{\partial}{\partial \tau_{i}}+\frac{i}{2} \frac{\partial}{\partial \chi_{i}}$.
} 
1. The volumes $t_{i}$ of a fixed basis of curves (two-cycles). These have the advantage that the geometrically allowed values form a cone, the Kähler cone.

2. The volumes $\tau_{i}$ of a fixed basis of divisors or, more generally, four-cycles. These are the standard choice of fields in the supergravity action.

Thanks to the Hodge-Riemann bilinear relations $\operatorname{det}\left(\frac{\partial \tau}{\partial t}\right)>0$ for all $\vec{t} \in \operatorname{Kc}(X)$. Therefore, the basis change $\left\{t_{i}\right\} \leftrightarrow\left\{\tau_{i}\right\}$ is nowhere singular inside the Kähler cone, and both sets of fields are good coordinates on the moduli space.

3. The "angular" variables $a_{i}$. By construction, they do not scale with the overall size of the manifold, see eq. (2.23). To parametrize the Kähler moduli space we need to pick a subset of $h_{+}^{11}-1$ variables $a_{i}$ plus one volume, which we take to be $V_{X}$.

\subsection{Minimizing the potential}

Let us start with $D_{i} W=0$, the condition for unbroken supersymmetry. Expanding the Kähler covariant derivative, one obtains

$$
\begin{aligned}
0=D_{i} W=\frac{\partial W}{\partial T_{i}}+W \frac{\partial K}{\partial T_{i}} & =-\frac{2 \pi}{N} n_{i} A e^{-\frac{2 \pi}{N} \vec{n} \cdot \vec{T}}+\frac{1}{2} K_{i}\left(W_{0}+A e^{-\frac{2 \pi}{N} \vec{n} \cdot \vec{T}}\right) \\
& =A e^{-\frac{2 \pi}{N} \vec{n} \cdot \vec{T}}(\underbrace{-\frac{2 \pi}{N} n_{i}+\frac{K_{i}}{2}}_{\in \mathbb{R}<}+\frac{W_{0} e^{\frac{2 \pi i}{N} \vec{n} \cdot \vec{\chi}}}{A} \underbrace{\frac{K_{i}}{2} e^{\frac{2 \pi}{N} \vec{n} \cdot \vec{\tau}}}_{\in \mathbb{R}_{<}}) .
\end{aligned}
$$

These are $h_{+}^{11}$ complex equations for the $2 h_{+}^{11}$ real variables $\left\{\tau_{i}, \chi_{i}\right\}$. Note that $K_{i}$ is real and negative, see eq. (2.20). Therefore, in order to cancel, the third summand must be real and positive, that is,

$$
\frac{1}{N} \vec{n} \cdot \vec{\chi}^{\text {susy }}+\frac{1}{2 \pi} \arg \left(\frac{W_{0}}{A}\right) \in \mathbb{Z}+\frac{1}{2} .
$$

In particular, we see that only the linear combination $\vec{n} \cdot \vec{\chi}$ gets fixed to a specific value; If $\frac{W_{0}}{A}$ has a complex phase then the axions dynamically adjust. For simplicity, we will take $W_{0}, A$ to be real and of opposite sign in the following, say, $W_{0}<0$ and $A>0$. Then one axion will be stabilized at

$$
\vec{n} \cdot \vec{\chi}^{\text {susy }}=0 \bmod \frac{N}{2 \pi} .
$$

In fact, this feature is highly desirable if one tries to use the axions in order to solve the strong $\mathrm{CP}$ problem in the context of string theory, and we will further discuss the phenomenological implications in subsection 5.3. Having taken care of the axions, we are left with $h_{+}^{11}$ real conditions for the variables $\left\{\tau_{i}\right\}$ or, equivalently, $\left\{t_{i}\right\}$. Using eq. (2.20), we obtain

$$
t_{i}^{\text {susy }}=n_{i} \frac{\frac{4 \pi}{N} V_{X}}{\left(-\frac{W_{0}}{A}\right) e^{\frac{2 \pi}{N} \vec{n} \cdot \vec{\tau}}-1} .
$$

We note that the supersymmetric minimum is achieved at $t_{i}^{\text {susy }} \sim n_{i}$ with a positive constant of proportionality. Therefore, the moduli are stabilized within the Kähler cone, see eq. (2.8), if and only if all $n_{i}>0$. This is why the (single) divisor $D$ contributing to the 
superpotential must be ample in order to stabilize the Kähler moduli with all volumes positive.

So far we used the F-terms, but in reality we want to minimize the scalar potential, eq. (2.12), with the added supersymmetry-violating term

$$
V=e^{K}\left(G^{i \bar{j}} \frac{\partial W}{\partial T_{i}} \frac{\overline{\partial W}}{\partial T_{j}}-3|W|^{2}\right)+\frac{D}{V_{X}^{r}} .
$$

This is where the third parametrization of the Kähler moduli in terms of, say, real coordinates $\left(a_{1}, a_{2}, \ldots, a_{h_{+}^{11}(X)-1}, V_{X}\right)$ plus their axionic partners becomes essential. Note that the supersymmetry-breaking term $D / V_{X}^{r}$ depends on $V_{X}$ only. Hence, the position of the minimum of the potential is unchanged as far as the remaining Kähler moduli $a_{i}$ as well as all axions are concerned. Therefore,

$$
\begin{aligned}
a_{i}^{\text {min }} & =a_{i}^{\text {susy }}=\frac{\tau_{i}\left(\vec{t}^{\text {susy }}\right) t_{i}^{\text {susy }}}{2 V_{X}\left(\vec{t}^{\text {susy }}\right)}=\frac{n_{i} \tau_{i}(\vec{n})}{2 V_{X}(\vec{n})}=n_{i} \frac{\partial}{\partial n_{i}} \ln \sqrt{\sum d_{j k \ell} n_{j} n_{k} n_{\ell}}, \\
\vec{n} \cdot \vec{\chi}^{\text {min }} & =\vec{n} \cdot \vec{\chi}^{\text {susy }}=0,
\end{aligned}
$$

see eqs. (2.13), (2.22) and (2.27). Only the "radial direction" of the Kähler moduli, parametrized by the volume $V_{X}$, is changed by the addition of the supersymmetry-breaking term. Moreover, having fixed the $a_{i}$, the volume $\tau_{D}=\vec{n} \cdot \vec{\tau}$ of the divisor $D$ is proportional to $V_{X}^{2 / 3}$ with a constant of proportionality

$$
\frac{\tau_{D}}{\left(V_{X}\right)^{\frac{2}{3}}}=\frac{\sum n_{i} \tau_{i}\left(\vec{t}^{\text {susy }}\right)}{V_{X}(\vec{t} \text { susy })^{\frac{2}{3}}}=\frac{\sum n_{i} \frac{\partial}{\partial n_{i}}\left(\sum d_{j k \ell} n_{j} n_{k} n_{\ell}\right)}{\left(\sum d_{j k \ell} n_{j} n_{k} n_{\ell}\right)^{\frac{2}{3}}}=3\left(\sum_{j k \ell} d_{j k \ell} n_{j} n_{k} n_{\ell}\right)^{\frac{1}{3}} .
$$

Therefore, minimizing the scalar potential for the $h_{+}^{11}$ fields $\left\{T_{i}\right\}$ boils down to minimizing it with respect to the single complexified Kähler modulus

$$
T_{D}=\tau_{D}+i \chi_{D}=(\vec{n} \cdot \vec{\tau})+i(\vec{n} \cdot \vec{\chi}),
$$

where we moreover already know that $\chi_{D}^{\text {susy }}=\chi_{D}^{\min }=0 \bmod \frac{N}{2 \pi}$, though we will not eliminate it quite yet. The scalar potential simplifies to

$$
\begin{aligned}
V & =\frac{1}{V_{X}^{2}\left(\tau_{D}\right)}\left[\left(\frac{1}{4} \frac{\partial^{2} K}{\partial \tau_{D}^{2}}\right)^{-1}\left|D_{T_{D}} W\right|^{2}-3|W|^{2}\right]+\frac{D}{V_{X}^{r}\left(\tau_{D}\right)} \\
& =\frac{1}{V_{X}^{2}\left(\tau_{D}\right)}\left[\frac{8 \pi}{N} \tau_{D} A e^{-\frac{2 \pi}{N} \tau_{D}}\left(\left(\frac{2 \pi}{3 N} \tau_{D}+1\right) A e^{-\frac{2 \pi}{N} \tau_{D}}+W_{0} \cos \left(\frac{2 \pi \chi_{D}}{N}\right)\right)+\frac{D}{V_{X}^{r-2}\left(\tau_{D}\right)}\right],
\end{aligned}
$$

where we used eqs. (2.20) and

$$
D_{T_{D}} W=\frac{1}{2}\left(D_{\tau_{D}} W-i D_{\chi_{D}} W\right)=-\frac{1}{2 \tau_{D}}\left(\left(\frac{4 \pi}{N} \tau_{D}+3\right) A e^{-\frac{2 \pi}{N}\left(\tau_{D}+i \chi_{D}\right)}+3 W_{0}\right) .
$$

Minimizing with respect to $\chi_{D}$, we immediately recognize that

$$
\frac{\partial V}{\partial \chi_{D}} \stackrel{!}{=} 0 \quad \Leftrightarrow \quad \sin \left(\frac{2 \pi \chi_{D}^{\min }}{N}\right)=0 \quad \Leftrightarrow \quad \chi_{D}^{\min }=\chi_{D}^{\text {susy }}=0 \quad \bmod \frac{N}{2 \pi}
$$


and the addition of the supersymmetry-breaking term does, indeed, not change the axions. Hence we set $\chi_{D}=0$ and proceed to minimize with respect to $\tau_{D}$,

$$
\frac{\partial V}{\partial \tau_{D}}=\frac{32 \pi}{3 N} \frac{\tau_{D}}{V_{X}^{2}}\left(\frac{\pi}{N} \tau_{D}+1\right)\left(\left.D_{T_{D}} W\right|_{T_{D}=\tau_{D}}\right) A e^{-\frac{2 \pi}{N} \tau_{D}}-\frac{3 r D}{2 \tau_{D} V_{X}^{r}} \stackrel{!}{=} 0 .
$$

As expected, if we set $D=0$ then supersymmetry is restored and the minimum of the scalar potential is given by the F-term $D_{T_{D}} W=0$. However, if we turn on $D \neq 0$, the stabilized volume $V_{D}^{\text {min }}$ is implicitly determined by the transcendental eq. (2.35), but cannot be solved for analytically. In the remainder of this section, we will compare it to the supersymmetric solution

$$
\left.0 \stackrel{!}{=}\left(D_{T_{D}} W\right)\right|_{T_{D}=\tau_{D}^{\text {susy }}} \quad \Leftrightarrow \quad \tau_{D}^{\text {susy }}=\frac{N}{2 \pi}\left[-\Omega_{-1}\left(\frac{3 W_{0}}{2 A} e^{-\frac{3}{2}}\right)-\frac{3}{2}\right],
$$

where we remind the reader that $W_{0} / A<0$ in our notation. Here ${ }^{11} \Omega_{-1}$ is the non-principal branch of the Lambert $\mathrm{W}$-function. It is real and negative on the domain

$$
-e^{-1}<\frac{3 W_{0}}{2 A} e^{-\frac{3}{2}}<0,
$$

with an essential singularity at 0 . Using the expansion

$$
\Omega_{-1}(x)=\ln (-x)-\ln (-\ln (-x))+\ldots
$$

around $x=0^{-}$, we obtain

$$
\tau_{D}^{\text {susy }}=\frac{N}{2 \pi}\left(\ln \left|\frac{2 A}{3 W_{0}}\right|+\ln \left(\frac{3}{2}+\ln \left|\frac{2 A}{3 W_{0}}\right|\right)+\ldots\right) .
$$

For the above solution to be valid, that is, to be in the regime in which the supergravity approximation holds, requires

$$
0<-\frac{3 W_{0}}{2 A} \ll 1 \Rightarrow\left|\frac{2 A}{3 W_{0}}\right| \gg 1 .
$$

Taking $A$ to be $\mathcal{O}(1)$, this automatically implies that the gravitino mass ${ }^{12} m_{3 / 2}=e^{K / 2} W \approx$ $W_{0}$ is much smaller than the Planck scale, ${ }^{13}$ and hence could give rise to low energy supersymmetry at around the $\mathrm{TeV}$ scale.

To estimate the effect of a small supersymmetry breaking term, we use eq. (2.36) to Taylor expand

$$
\left.\frac{\partial V}{\partial \tau_{D}}\right|_{\tau_{D}=\tau_{D}^{\text {susy }}+\epsilon}=\frac{-3 r D}{2 \tau_{D}^{\text {susy }} V_{X}^{r}\left(\tau_{D}^{\text {susy }}\right)}+12 \frac{\frac{8 \pi^{2}}{N^{2}}\left(\frac{\pi}{N} \tau_{D}^{\text {susy }}+1\right)\left(\frac{4 \pi}{N} \tau_{D}^{\text {susy }}+1\right)\left(\tau_{D}^{\text {susy }} W_{0}\right)^{2}}{\left(\frac{4 \pi}{N} \tau_{D}^{\text {susy }}+3\right)^{2}\left(\tau_{D}^{\text {susy }}\right)^{2} V_{X}^{2}\left(\tau_{D}^{\text {susy }}\right)} \epsilon+O\left(\epsilon D, \epsilon^{2}\right) .
$$

Therefore, to first order, the minimum of the scalar potential is at

$$
\tau_{D}^{\min }=\tau_{D}^{\text {susy }}+\frac{r N^{2}}{64 \pi^{2}} \frac{\left(\frac{4 \pi^{2}}{N^{2}} \tau_{D}^{\text {susy }}+3\right)^{2}}{\left(\frac{\pi}{N} \tau_{D}^{\text {susy }}+1\right)\left(\frac{4 \pi}{N} \tau_{D}^{\text {susy }}+1\right) \tau_{D}^{\text {susy }} W_{0}^{2} V_{X}^{r-2}\left(\tau_{D}^{\text {susy }}\right)} D+O\left(D^{2}\right) .
$$

To summarize, we have found that:

\footnotetext{
${ }^{11}$ We will use $\Omega$ instead of $W$ for the Lambert $W$-function to differentiate it from the superpotential.

${ }^{12}$ This is true if the volume $V_{X}$ is consistent with standard gauge unification, i.e. it is not too large.

${ }^{13}$ Here we set $m_{\mathrm{Pl}}=1$.
} 
- The real part of the Kähler moduli are stabilized inside the Kähler cone, that is, with all volumes positive.

- One axion is stabilized, the remaining $h_{+}^{11}-1$ remain massless.

- The only effect of the supersymmetry-breaking addition to the scalar potential is to increase the overall volume at which the moduli are fixed. Neither the axions nor the angular part of the Kähler moduli is changed.

Finally, before moving on to providing explicit examples of Calabi-Yau orientifolds, which realize the above mechanism, it is worthwhile to comment on the size of the explicit supersymmetry breaking contribution due to anti D3-branes. As mentioned earlier, these positive contributions to the potential can give rise to a de Sitter vacuum under certain conditions, the same as in the KKLT proposal. These contributions are exponentially small due to strong warping at the bottom of the warped throat where these anti D3-branes are dynamically trapped, leading to $0<D \ll 1$. Therefore, to obtain a de Sitter vacuum this requires the tree-level superpotential contribution $W_{0}$ to be exponentially small as well since otherwise the uplifting would not be strong enough and the vacuum would remain AdS. Furthermore, in our analysis we have focused on Kahler moduli only assuming that the complex structure moduli and the dilaton are stabilized by fluxes at a much higher scale [58]. As argued in [47, 48], the decoupling between the dynamics of Kahler moduli and complex structure moduli plus the dilaton is only justified when $W_{0}$ is small enough. ${ }^{14}$ Thus, we see that the requirement of a small $W_{0} \ll 1$ has four separate origins:

- Moduli Stabilization in the supergravity regime.

- Decoupling of complex structure moduli and the dilaton.

- A small gravitino mass relative to the Planck scale.

- Realizing a de Sitter Vacuum.

As mentioned earlier, although one naturally expects $W_{0}=\mathcal{O}(1)$, by choosing appropriate values of the integer fluxes it is possible to tune $W_{0} \ll 1$, see $[51,52]$. Then, as we will explicitly see in subsubsection 3.2.3, one can actually arrange the numerical values to yield a de Sitter vacuum with a small cosmological constant.

\section{$3 \quad$ Explicit examples}

In this section, we provide some explicit examples of Calabi-Yau orientifolds. To start with, we will consider a simple case with just one Kähler modulus and construct a divisor which is both rigid and ample, thereby contributing to the superpotential. We then consider the relevant case with three Kähler moduli in which we realize the mechanism outlined in section 2 .

\footnotetext{
${ }^{14}$ The very large volume scenario [11] where $W_{0} \sim \mathcal{O}(1)$ presents an exception because in that case the Kahler potential is also large due to the exponentially large volume.
} 


\subsection{Simple case - a one-parameter model}

The simplest way for D3-instantons to stabilize the Kähler moduli in the interior of the Kähler cone is to have a single instanton wrapped on a rigid ample divisor. Recall from subsection 2.1 that this automatically guarantees that $\chi_{+}-\chi_{-}=1$ and, therefore, the divisor contributes to the superpotential.

The starting point for our example is the $\mathbb{P}^{7}[2222]$ complete intersection Calabi-Yau manifold, that is, the complete intersection of 4 quadrics in $\mathbb{P}^{7}$. There is a free $\mathbb{Z}_{2} \times \mathbb{Z}_{2} \times \mathbb{Z}_{2^{-}}$ action on $\widetilde{X}[21,23,24,28,57]$. We will always divide out this group action and define

$$
X=\widetilde{X} /\left(\mathbb{Z}_{2} \times \mathbb{Z}_{2} \times \mathbb{Z}_{2}\right) .
$$

The Hodge numbers of the CICY threefold $\widetilde{X}$ are well-known. Since the $\left(\mathbb{Z}_{2}\right)^{3}$ action necessarily preserves the Kähler class, we obtain

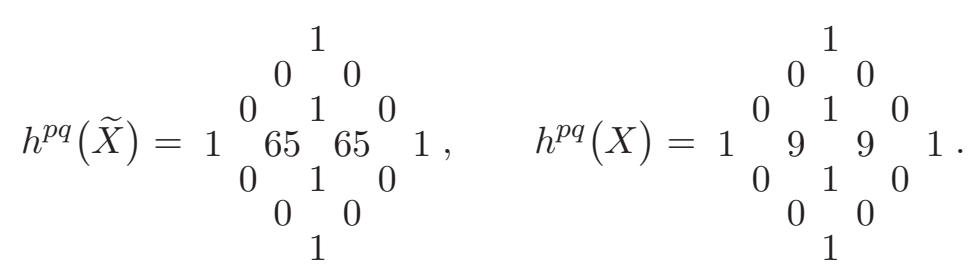

The group action on the homogeneous coordinates $z_{0}, \ldots, z_{7}$ of $\mathbb{P}^{7}$ is the regular representation. Explicitly, it is generated by

$$
\begin{aligned}
& g_{1}=\operatorname{diag}(+1,+1,+1,+1,-1,-1,-1,-1), \\
& g_{2}=\operatorname{diag}(+1,+1,-1,-1,+1,+1,-1,-1), \\
& g_{3}=\operatorname{diag}(+1,-1,+1,-1,+1,-1,+1,-1) .
\end{aligned}
$$

A basis for the $\left(\mathbb{Z}_{2}\right)^{3}$-invariant polynomials is spanned by $p_{i}=z_{i}^{2}, 0 \leq i \leq 7$. One can check [54] that the zero set of four generic linear combinations of invariant degree2 polynomials is a smooth Calabi-Yau threefold $\widetilde{X}$, and that the $\left(\mathbb{Z}_{2}\right)^{3}$ action on $\widetilde{X}$ is fixed-point free. Therefore, the quotient $X$ is again a smooth Calabi-Yau threefold.

We now consider the line bundle $\mathscr{O}_{\widetilde{X}}(1)$, whose sections are

$$
H^{0}\left(\widetilde{X}, \mathscr{O}_{\widetilde{X}}(1)\right)=\operatorname{span}_{\mathbb{C}}\left\{z_{0}, \ldots, z_{7}\right\},
$$

and all higher-degree cohomology groups vanish. Decomposing this representation into irreducible representations, we find a unique invariant section

$$
H^{0}\left(X, \mathscr{O}_{X}(1)\right)=H^{0}\left(\widetilde{X}, \mathscr{O}_{\widetilde{X}}(1)\right)^{\mathbb{Z}_{2}^{3}}=\mathbb{C} \cdot z_{0} .
$$

We denote the corresponding divisor by

$$
\widetilde{D}=\left\{z_{0}=0\right\} \subset \widetilde{X}, \quad D=\widetilde{D} /\left(\mathbb{Z}_{2}^{3}\right) \subset X .
$$

The Hodge numbers of the divisors are related to the line bundle cohomology by

$$
h^{00}(D)=h^{2}(X, \mathscr{O}(D))+1, \quad h^{01}(D)=h^{1}(X, \mathscr{O}(D)), \quad h^{02}(D)=h^{0}(X, \mathscr{O}(D))-1 .
$$

They enjoy the following properties: 
- Both $\widetilde{D}, D$ are ample because $\mathscr{O}(1)$ is an ample line bundle.

- $D$ is rigid by eq. (3.5).

- Using [54], one can check that $D$ is a smooth complex surface for generic complex structure moduli of $X$.

Therefore, $D$ satisfies the sufficient requirements to contribute to the superpotential.

Let us further investigate the geometry of the divisor $D$. A standard computation yields the Chern numbers. By abuse of notation, we denote by $h$ the hyperplane classes in $\mathbb{P}^{7}$ as well as its restriction to $\tilde{X}, \widetilde{D}$. Using this notation,

$$
\begin{aligned}
& c_{1}(\tilde{X})=-K_{\tilde{X}}=0, \quad c_{2}(\tilde{X})=4 h^{2}, \quad c_{3}(\widetilde{X})=-8 h^{3}, \\
& c_{1}(\widetilde{D})=-K_{\widetilde{D}}=-h, \quad c_{2}(\widetilde{D})=5 h^{2} .
\end{aligned}
$$

It is now easy to compute the Chern numbers, and we obtain

$$
\begin{aligned}
\int c_{1}(\widetilde{D})^{2} & =16 \quad \Rightarrow \int c_{1}(D)^{2}=2, \\
\chi(\widetilde{D})=\int c_{2}(\widetilde{D})=80 & \Rightarrow \int c_{2}(D)=10=\chi(D) .
\end{aligned}
$$

Using the Euler number, and the above properties of the divisors, it is easy to determine their Hodge numbers,

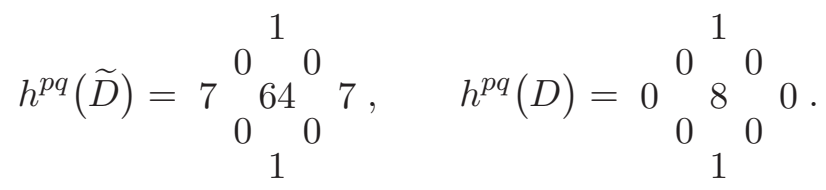

We observe that $D$ is a numerical Campedelli surface $[12,63]$ with $\pi_{1}(D)=\mathbb{Z}_{2} \times \mathbb{Z}_{2} \times \mathbb{Z}_{2}$.

In fact, the appearance of this surface is not a coincidence. By adjunction, an ample divisor in a Calabi-Yau threefold is a complex surface with ample canonical ${ }^{15}$ bundle. Such a surface is called of general type, and can be roughly classified by the Chern numbers $c_{1}^{2}=\int c_{1}(D)^{2}$ and $c_{2}=\int c_{2}(D)$. If we now furthermore impose the arithmetic genus $\chi\left(D, \mathscr{O}_{D}\right)=1$, then Hirzebruch-Riemann-Roch yields

$$
1=\chi\left(D, \mathscr{O}_{D}\right)=\int \operatorname{ch}\left(\mathscr{O}_{D}\right) \operatorname{Td}(T D)=\int \frac{c_{1}(D)^{2}+c_{2}(D)}{12} .
$$

Since $c_{1}^{2}, c_{2}>0$ for surfaces of general type, this leaves us with 11 possibilities. The Bogomolov-Miyaoka-Yau inequality $c_{1}^{2} \leq 3 c_{2}$ excludes two.

We list a number of examples in table 1. Note that the surfaces arising as ample divisors in Calabi-Yau manifolds have $\left|\pi_{1}(D)\right|=\left|\pi_{1}(X)\right|<\infty$, which further restricts their Chern numbers to $1 \leq c_{1}^{2} \leq 5$.

So far, we have not specified the orientifold involution. The obvious choice is to pick one homogeneous coordinate $z_{j}$ and send $z_{j} \mapsto-z_{j}$. This is a well-defined orientifold action on $X$ because $\tilde{X}$ is invariant and the orientifold action commutes with the $\left(\mathbb{Z}_{2}\right)^{3}$-action. The fixed-point set is a single O7-plane. We distinguish the following two cases:

\footnotetext{
${ }^{15}$ Not to be confused with del Pezzo surfaces, whose anti-canonical bundle is ample.
} 


\begin{tabular}{|cc|cll|}
\hline$c_{1}^{2}$ & $c_{2}$ & $\left|\pi_{1}(D)\right|<\infty$ & Example & Ambient Calabi-Yau \\
\hline 1 & 11 & Yes & Godeaux surface & Quintic $/ \mathbb{Z}_{5}$ \\
2 & 10 & Yes & Campedelli surfaces & CICY $\# 7884 /\left(\mathbb{Z}_{3} \times \mathbb{Z}_{3}\right)$ \\
3 & 9 & Yes & Burniat surfaces & CICY $\left.\# 7862 /\left(\mathbb{Z}_{2} \times Q_{8}\right)\right)$ \\
4 & 8 & Yes & Burniat surfaces & CICY \#7861/( $\left.\mathbb{Z}_{8} \rtimes \mathbb{Z}_{4}\right)$ \\
5 & 7 & Yes & Burniat surfaces & $?$ \\
6 & 6 & No & Burniat surfaces & N/A \\
7 & 5 & No & Inoue surface & N/A \\
8 & 4 & No & Beauville surface & N/A \\
9 & 3 & No & fake $\mathbb{P}^{2}$ & N/A \\
\hline
\end{tabular}

Table 1. Examples of surfaces of general type with $\chi\left(D, \mathscr{O}_{D}\right)=1$ and how they arise as rigid ample divisors in Calabi-Yau threefolds.

- $j=0$ : $D$ sits on top of ${ }^{16}$ the $O 7$.

- $j \neq 0$ : $D$ intersects the $O 7$-plane in a genus-3 curve.

In either case, a D3-instanton wrapped on the divisor $D$ satisfies the necessary criteria to contribute to the superpotential.

\section{$3.2 \quad$ A three-parameter model}

\subsubsection{The geometry}

We now consider a particular example of a Calabi-Yau threefold with $h^{11}=h^{21}=19$. It contains an ample divisor $D$ with $\vec{n}=\{1,1,1\}$ such that $\chi_{+}-\chi_{-}=1$. Note that $D$ is not rigid, yet still satisfies the necessary condition to contribute to the superpotential. We start with the CICY \#18,

$$
\begin{aligned}
& \mathbb{P}^{1} \\
& \mathbb{P}^{2} \\
& \mathbb{P}^{3}\left[\begin{array}{lll}
0 & 1 & 1 \\
0 & 0 & 3 \\
3 & 1 & 0
\end{array}\right] .
\end{aligned}
$$

Now, define the orientifold $\mathbb{Z}_{2}$-action to be generated by

$$
\Omega\left(\left[x_{0}: x_{1}\left|y_{0}: y_{1}: y_{2}\right| z_{0}: z_{1}: z_{2}: z_{3}\right]\right)=\left[x_{0}:-x_{1}\left|y_{0}:-y_{1}:-y_{2}\right| z_{0}:-z_{1}:-z_{2}:-z_{3}\right]
$$

and demand that the polynomials (of the required multi-degrees) transform as

$$
p_{(0,0,3)} \circ \Omega=-p_{(0,0,3)}, \quad p_{(1,0,1)} \circ \Omega=p_{(1,0,1)}, \quad p_{(1,3,0)} \circ \Omega=p_{(1,3,0)} .
$$

\footnotetext{
${ }^{16}$ The instanton sitting on top of an $O 7^{(-)}$orientifold plane would carry $\mathrm{Sp}(2)$ gauge group. For this to contribute to the superpotential, a suitable flux or intersections with further branes would be necessary to lift fermion zero modes. Alternatively, one could use a $O 7^{(+)}$plane. Of course, within a 1-parameter model it is then not possible to cancel the $D 7$ tadpole. In any case, further input about physical objects beyond the background geometry would be needed to decide whether the instanton definitely contributes to the superpotential.
} 


\begin{tabular}{|c|c|c|c|c|c|c|c|c|}
\hline $\mathbb{P}^{1}$ & $\mathbb{P}^{2}$ & $\mathbb{P}^{3}$ & 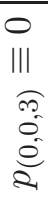 & $\begin{array}{c}0 \\
\text { III } \\
\text { ? } \\
0 \\
2 \\
2\end{array}$ & 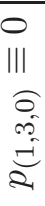 & $\begin{array}{l}0 \\
\text { III } \\
= \\
= \\
= \\
=\end{array}$ & O-planes & O-planes $\cap D$ \\
\hline$[1: 0]$ & {$[0: *: *]$} & {$[0: *: *: *]$} & $\mathrm{N}$ & $\mathrm{Y}$ & Y & $\mathrm{N}$ & $\mathrm{O} 7$ & $T^{2}$ \\
\hline$[0: 1]$ & {$[0: *: *]$} & {$[0: *: *: *]$} & $\mathrm{N}$ & $\mathrm{N}$ & $\mathrm{N}$ & $\mathrm{Y}$ & Nine O3 & 9 points \\
\hline$[1: 0]$ & {$[1: 0: 0]$} & {$[0: *: *: *]$} & $\mathrm{N}$ & $\mathrm{Y}$ & $\mathrm{N}$ & $\mathrm{Y}$ & - & - \\
\hline$[0: 1]$ & {$[1: 0: 0]$} & {$[0: *: *: *]$} & $\mathrm{N}$ & $\mathrm{N}$ & Y & $\mathrm{N}$ & Three O3 & - \\
\hline$[1: 0]$ & {$[1: *: *]$} & {$[1: 0: 0: 0]$} & $\mathrm{Y}$ & $\mathrm{N}$ & Y & $\mathrm{Y}$ & - & - \\
\hline$[0: 1]$ & {$[1: *: *]$} & {$[1: 0: 0: 0]$} & $\mathrm{Y}$ & $\mathrm{Y}$ & $\mathrm{N}$ & $\mathrm{N}$ & Three O3 & - \\
\hline$[1: 0]$ & {$[1: 0: 0]$} & {$[1: 0: 0: 0]$} & $\mathrm{Y}$ & $\mathrm{N}$ & $\mathrm{N}$ & $\mathrm{N}$ & - & - \\
\hline$[0: 1]$ & {$[1: 0: 0]$} & {$[1: 0: 0: 0]$} & $\mathrm{Y}$ & $\mathrm{Y}$ & Y & Y & O3 & 1 point \\
\hline
\end{tabular}

Table 2. Orientifold planes on $X \subset \mathbb{P}^{1} \times \mathbb{P}^{2} \times \mathbb{P}^{3}$ arising from different patches of the ambient space. Geometrically, the O7-plane spans $\mathbb{R}^{3,1} \times \mathbb{P}^{1} \times T^{2}$.

Note that, although these polynomials are not invariant, their zero set is. Using [54], one can check that generic such polynomials cut out a smooth ${ }^{17}$ Calabi-Yau threefold $X=\left\{p_{(0,0,3)}=p_{(1,0,1)}=p_{(1,3,0)}=0\right\}$. Moreover, a generic $\mathbb{Z}_{2}$-invariant polynomial

$$
p_{(1,1,1)} \in H^{0}(X, \mathscr{O}(1,1,1))^{\mathbb{Z}_{2}}
$$

defines a smooth divisor $D=\left\{p_{(1,1,1)}=0\right\} \subset X$, which we will take to be our D3-instanton. Using the Koszul spectral sequence, one can compute that

$$
\chi_{+}\left(D, \mathscr{O}_{D}\right)=11, \quad \chi_{-}\left(D, \mathscr{O}_{D}\right)=10 .
$$

The divisor $D$ is ample, and therefore is a surface of general type with $\pi_{1}(D)=\pi_{1}(X)=1$. Its Chern numbers are $c_{1}^{2}=90, c_{2}=162$. It intersects the O7-plane in a smooth elliptic curve as well as 10 out of the 16 O3-planes, see table 2. Therefore, the Hodge numbers of $D$ are

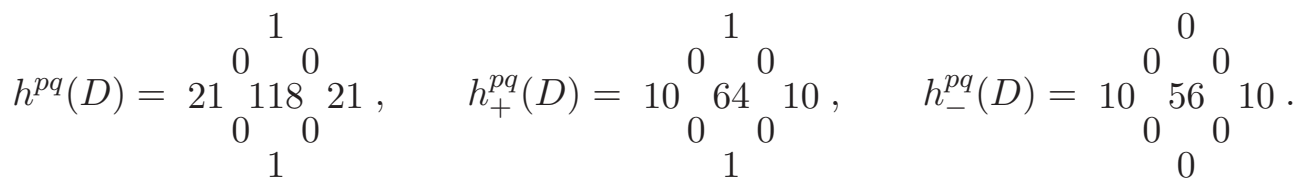

The Calabi-Yau threefold has Hodge numbers $h^{11}(X)=19=h^{21}(X)$, but only a 3 dimensional sublattice of $H_{4}(X, \mathbb{Z}) \simeq \mathbb{Z}^{19}$ is spanned by the divisors $h_{1}=\left\{x_{0}=0\right\}$, $h_{2}=\left\{y_{0}=0\right\}, h_{3}=\left\{z_{0}=0\right\}$. As long as we are only considering instantons wrapped on

\footnotetext{
${ }^{17}$ We remark that eq. (3.13) is the unique action that admits a smooth embedded threefold.
} 
divisors $D$ in this sublattice, we can consistently ignore the remaining $19-3=16$ Kähler moduli. Therefore, we parametrize the Kähler class as $\omega=t_{1} h_{1}+t_{2} h_{2}+t_{3} h_{3}$. The volumes of the relevant (sub-)manifolds are ${ }^{18}$

$$
\begin{gathered}
V_{X}=\frac{3}{2} t_{2} t_{3}\left(6 t_{1}+t_{2}+3 t_{3}\right), \\
\tau_{1}=\frac{\partial V_{X}}{\partial t_{1}}=\operatorname{Vol}\left(h_{1}\right)=9 t_{2} t_{3}, \\
\tau_{2}=\frac{\partial V_{X}}{\partial t_{2}}=\operatorname{Vol}\left(h_{2}\right)=\frac{3}{2} t_{3}\left(6 t_{1}+2 t_{2}+3 t_{3}\right), \\
\tau_{3}=\frac{\partial V_{X}}{\partial t_{3}}=\operatorname{Vol}\left(h_{3}\right)=\frac{3}{2} t_{2}\left(6 t_{1}+t_{2}+6 t_{3}\right), \\
\tau_{D}=\vec{n} \cdot \vec{\tau}=\frac{9}{2} t_{3}^{2}+\frac{3}{2} t_{2}^{2}+9 t_{1} t_{2}+9 t_{1} t_{3}+21 t_{2} t_{3} .
\end{gathered}
$$

In these coordinates, the Kähler cone is precisely the first octant $t_{1}, t_{2}, t_{3}>0$.

\subsubsection{Stabilization of moduli}

Armed with an expression for the volume in terms of the two-cycles $t_{i}$, and that for the $t_{i}$ in terms of the $\tau_{i}$ in eq. (3.18), we can express the volume $V_{X}$ in terms $\tau_{1}, \tau_{2}$ and $\tau_{3}$. As we remarked earlier, the coordinate change is one-to-one inside the Kähler cone. However, since the $\tau_{i}$ are quadratic in the $t_{i}$, some non-physical values of the $t_{i}$ are also mapped to allowed values of the $\tau_{i}$. Hence, when we invert the quadrics, care must be taken to choose the correct branch for each root. The unique solution for the two-cycles in terms of the four-cycles is

$$
t_{1}=\frac{4\left(3 \tau_{2}-\tau_{1}\right) \tau_{3}-3 \tau_{1}\left(4 \tau_{2}-\tau_{1}\right)}{6 \sqrt{3} \sqrt{\tau_{1}\left(6 \tau_{2}-\tau_{1}\right)\left(2 \tau_{3}-\tau_{1}\right)}}, \quad t_{2}=\frac{\sqrt{\tau_{1}\left(2 \tau_{3}-\tau_{1}\right)}}{\sqrt{3} \sqrt{\left(6 \tau_{2}-\tau_{1}\right)}}, \quad t_{3}=\frac{\sqrt{\tau_{1}\left(6 \tau_{2}-\tau_{1}\right)}}{3 \sqrt{3} \sqrt{\left(2 \tau_{3}-\tau_{1}\right)}}
$$

Then, the Kähler potential equals

$$
K=-2 \ln \left(\frac{3}{2} t_{2} t_{3}\left(6 t_{1}+t_{2}+3 t_{3}\right)\right)=-2 \ln \left(\frac{1}{6 \sqrt{3}} \sqrt{\tau_{1}\left(6 \tau_{2}-\tau_{1}\right)\left(2 \tau_{3}-\tau_{1}\right)}\right) .
$$

There exists an ample divisor satisfying the criterion $\chi_{+}-\chi_{-}=1$, whose volume is given by

$$
\tau_{D}=\tau_{1}+\tau_{2}+\tau_{3}
$$

that is, $n_{1}=n_{2}=n_{3}=1$. Therefore, we can now directly apply the methods developed in the previous subsection. The "angular" part of the moduli vevs is

$$
a_{1}^{\min }=a_{1}^{\text {susy }}=\frac{6}{20}, \quad a_{2}^{\min }=a_{2}^{\text {susy }}=\frac{11}{20}, \quad a_{3}^{\min }=a_{3}^{\text {susy }}=\frac{13}{20},
$$

using eq. (2.29).

The two alternative choices for coordinates on the moduli space, $\left\{t_{i}\right\}$ and $\left\{\tau_{i}\right\}$, are stabilized in the directions $t_{i} \sim n_{i}$ and $\tau_{i} \sim \frac{a_{i}}{n_{i}}$ respectively, see eqs. (2.27) and (2.22). The

\footnotetext{
${ }^{18}$ Note that, as an abstract cubic polynomial in three variables, $V_{X}$ has discriminant $\Delta=0$. This proves that no coordinate change can bring it into the swiss cheese form, which would have $\Delta \neq 0$.
} 
constant of proportionality can be parametrized by one volume, which we take to be $\tau_{D}$. Using eq. (2.13) and $\tau_{D}=\vec{n} \cdot \vec{\tau}$, we can fix the constant of proportionality in general to be

$$
t_{i}=\left(\frac{V_{X}}{\sum d_{j k \ell} n_{j} n_{k} n_{\ell}}\right)^{\frac{1}{3}} n_{i}, \quad \tau_{i}=\frac{2}{3} \frac{a_{i}}{n_{i}} \tau_{D} .
$$

For the case at hand, we obtain

$$
\tau_{1}=\frac{6}{30} \tau_{D}, \quad \tau_{2}=\frac{11}{30} \tau_{D}, \quad \tau_{3}=\frac{13}{30} \tau_{D}, \quad t_{1}=t_{2}=t_{3}=\frac{\sqrt{\tau_{D}}}{3 \sqrt{5}}, \quad V_{X}=\frac{\tau_{D}^{3 / 2}}{9 \sqrt{5}},
$$

using eq. (3.19) and (2.30).

Note that in this explicit example all the four- and two-cycle volumes as well as the volume of the Calabi-Yau are functions of a single parameter - the volume $\tau_{D}$ of the ample divisor, which is determined by $W_{0}, A, N$ and $D$, and is in principle computable from the choice of fluxes and anti-D3 branes. This implies that in a compactification with a realistic visible sector, the volume of the visible sector four-cycle, which measures the gauge coupling at the compactification $(\mathrm{KK})$ scale is also fixed in terms of $\tau_{D}$. Thus, choosing phenomenologically well motivated values of $\tau_{\text {visible }}$ at $M_{K K} \sim M_{G U T}$, such as $\tau_{\text {visible }} \approx \alpha_{G U T}^{-1} \approx 25$, completely determines the values of all Kähler moduli, in particular the volume of the Calabi-Yau manifold! Furthermore, it provides a bottom-up constraint on the microscopic parameters $W_{0}, A, N$, and $D$. Of course, the complex structure moduli and the dilaton, whose vevs are controlled by the fluxes remain far less constrained. ${ }^{19}$

\subsubsection{Supersymmetry breaking and a particular choice of parameters}

As a concrete numerical example, consider the following choice of input parameters for the three parameter model considered in subsection 3.2

$$
A=1, \quad W_{0}=-10^{-13}, \quad N=10, \quad D=3.9 \times 10^{-27}, \quad r=\frac{4}{3} .
$$

Here we have assumed the origin of the non-perturbative superpotential to be a gaugino condensate with a dual Coxeter number $N=10$ as this helps in stabilizing the moduli in the supergravity regime. Also, as mentioned earlier, both $W_{0}$ and $D$ are required to be extremely small for the solution to be self-consistent and be phenomenologically viable. The scalar potential in the radial direction is plotted in figure 1. Numerically solving the transcendental equation eq. (2.35) in combination with eq. (3.24), we determine the minimum of the scalar potential at

$$
\tau_{D}^{\min } \approx 52.68 \Rightarrow \tau_{1}^{\min } \approx 10.54, \quad \tau_{2}^{\min } \approx 19.32, \quad \tau_{3}^{\min } \approx 22.83 .
$$

To verify the above result we also performed a numerical minimization of the scalar potential eq. (2.12) in the 3-dimensional Kähler moduli space using Mathematica for the same choice of input parameters. We again found a metastable de Sitter minimum with

$$
\tau_{1}^{\text {num }} \approx 10.54, \quad \tau_{2}^{\text {num }} \approx 19.32, \quad \tau_{3}^{\text {num }} \approx 22.83 .
$$

\footnotetext{
${ }^{19}$ In contrast, consider the fluxless $G_{2}$ compactifications of $M$ theory [4]. Here, one actually constrains all moduli in terms of a single parameter - the volume $V_{\mathcal{Q}}$ of an associative three-cycle $\mathcal{Q}$ that is Poincaré dual to the co-associative four form.
} 


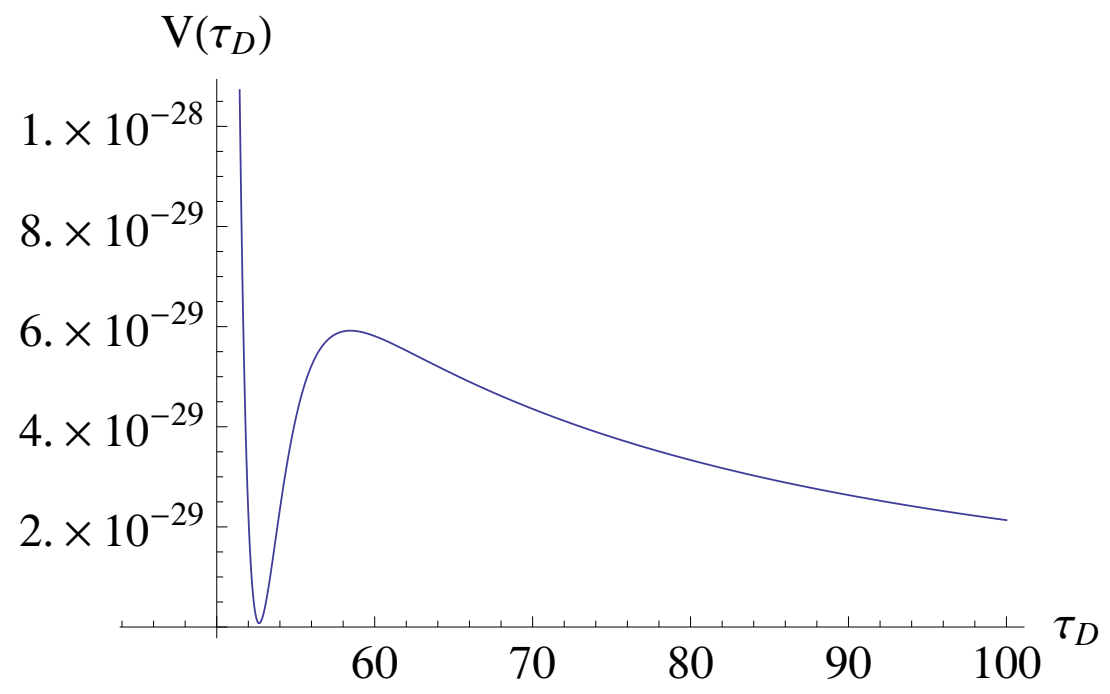

Figure 1. The scalar potential in the $\tau_{D}$-direction for the parameter choice in eq. (3.25).

The gravitino mass and the cosmological constant at the minimum are given by:

$$
m_{3 / 2}=e^{K / 2}|W| \approx 12 \mathrm{TeV}, \quad V\left(\tau_{D}^{\min }\right) \approx 7.4 \times 10^{-31} m_{\mathrm{Pl}}^{4}
$$

where we have absorbed the overall factor $e^{\mathcal{K} / 2}$ coming from the complex structure and the dilaton parts of Kähler potential into the effective parameters $W_{0}$ and $A$. Note that the vacuum energy, while huge compared to the actual value, is small in Planck units. Therefore, much smaller values can be reliably attained by a further tuning of $W_{0}, A$ and $D$, without affecting the moduli vevs and the gravitino mass as well as all phenomenologically relevant parameters such as the masses of superpartners. The feasibility of such tuning by fluxes is explained by the Bousso-Polchinski mechanism [22].

Let us now compute the Kähler moduli and axion spectrum. The three canonically normalized moduli eigenstates have masses

$$
m_{\Psi_{1}} \approx 65.67 m_{3 / 2} \approx 804.5 \mathrm{TeV}, \quad m_{\Psi_{2}}=m_{\Psi_{3}} \approx 1.34 m_{3 / 2} \approx 16.5 \mathrm{TeV}
$$

The heavy eigenstate $\Psi_{1}$ corresponds mostly to the breathing mode of the divisor volume $\tau_{D}$ while the modes $\Psi_{2}$ and $\Psi_{3}$ are mostly volume preserving. On the other hand, since the superpotential contains only one linear combination of the axions, only one of the axions receives a mass while two of the remaining eigenstates remain massless. Indeed, we find that the canonically normalized axion eigenstates have masses

$$
m_{\Phi_{1}} \approx 66.63 m_{3 / 2} \approx 816.3 \mathrm{TeV}, \quad m_{\Phi_{2}}=m_{\Phi_{3}}=0 .
$$

The flat directions $\Phi_{2,3}$ are the Goldstone bosons arising from the two shift symmetries that leave the linear combination $\chi_{1}+\chi_{2}+\chi_{3}$ invariant. It is important to realize, however, that the superpotential eq. (2.6) must be regarded as the dominant contribution in a series of terms with other highly subdominant non-perturbative contributions. These subdominant 
contributions will eventually fix the remaining axions, albeit at vastly suppressed scales. We will comment more on this in section 5 .

Finally, after rotating $\tilde{K}_{i} \equiv U_{i k}^{\dagger} K_{k l} U_{l j}, U \in \mathrm{U}(3)$, into the axion mass eigenstate basis the axion decay constants are given by

$f_{\Phi_{i}}=\sqrt{2 \widetilde{K}_{i}} m_{\mathrm{Pl}} \Rightarrow f_{1} \approx 1.7 \times 10^{17} \mathrm{GeV}, \quad f_{2} \approx 9.8 \times 10^{16} \mathrm{GeV}, \quad f_{3} \approx 9.1 \times 10^{16} \mathrm{GeV}$,

which is expected in string compactifications with a high string scale $M_{s} \gtrsim M_{G U T}$.

\section{Consistency condition for the single condensate/instanton approxima- tion}

As mentioned earlier, the superpotential eq. (2.6) must be regarded as the leading term in a series of contributions. In the presence of other contributions, it is important to show that the truncation to the superpotential can be made parametrically self-consistent. In order to illustrate that, let us assume that the superpotential contains two non-perturbative terms such that

$$
W=W_{0}-A e^{-\frac{2 \pi}{N} \sum_{i=1}^{h_{+}^{11}} n_{i} \tau_{i}}+B e^{-\frac{2 \pi}{M} \sum_{i=1}^{h_{+}^{11}} m^{i} \tau_{i}}
$$

where we have fixed the axions so that $W$ is real. Substituting the moduli vevs from eqs. (3.23) and (2.42), we obtain

$$
W=W_{0}-A e^{-\frac{2 \pi}{N} \tau_{D}}+B e^{-\frac{2 \pi}{M} \tau_{D}\left(\frac{2}{3} \sum_{i=1}^{h_{+}^{11}} \frac{m_{i} a_{i}}{n_{i}}\right)} .
$$

If the divisor contributing to the second term in the superpotential is a combination of basis four-cycles, then one expects $m_{i} \sim n_{i} \sim \mathcal{O}(1)$, which implies $\frac{2}{3} \sum \frac{m^{i} a_{i}}{n_{i}} \sim \mathcal{O}(1)$. Substituting an approximate expression for the divisor volume $\tau_{D} \approx \frac{N}{2 \pi} \ln \left|2 A / 3 W_{0}\right|$ into eq. (4.2) we get the following approximate expression for the superpotential at the minimum:

$$
W \approx W_{0}-\frac{3}{2} W_{0}+B\left|\frac{3 W_{0}}{2 A}\right|^{\frac{N}{M} \times \mathcal{O}(1)} .
$$

Therefore, when $N / M>\mathcal{O}(1)$, the extra condensates/instantons become exponentially suppressed relative to the leading contribution. It may also happen that some of the divisors contributing to the superpotential correspond to the basis four-cycles $\tau_{i}$, e.g. $m_{i}=$ $(0, \ldots, 1, \ldots, 0)$. In this case, because $a_{i} \sim \mathcal{O}\left(1 / h_{+}^{11}\right)$, we need $N>M \times h_{+}^{11} \gg M$ so that these contributions do not affect the moduli vevs and only help to fix the remaining axions.

The above mechanism is qualitatively different from the one when there are many (such as $h_{+}^{11}$ ) comparable terms in the superpotential. A natural setup in which this may occur is when the leading contribution comes from a condensing gauge group with a dual Coxeter number $N=\mathcal{O}(10-100)$ while the truncated terms arise from instantons (that is, $M=1$ ). Likewise, possible non-perturbative corrections to the Kähler potential from non-BPS instantons, which may also play an important role in stabilizing the remaining 
axions, can be made exponentially suppressed in a similar way. The single condensate approximation, therefore, can be naturally made parametrically self-consistent and should be quite robust.

\section{Phenomenological consequences}

\subsection{A chiral spectrum}

The first step in a phenomenologically viable construction is to get a realistic spectrum. As discussed in the introduction, in a realistic Type IIB compactification one is faced with the challenge of stabilizing the divisors whose intersections support charged chiral matter [20]. In particular, since an ample divisor $D$ automatically intersects all four cycles one may worry that due to the presence of extra zero modes the corresponding non-perturbative term is multiplied by a combination of visible sector chiral matter fields, whose vevs are known to be zero. This would certainly be true if one specifically selected the intersection curve $\Sigma=D \cap D_{\text {vis }}$, where the divisor $D_{\text {vis }}$ is wrapped by the visible sector D7 stack, as the one that supports charged chiral matter by turning on gauge flux (a non-trivial line bundle) along $\Sigma$. However, we see no a priori reason for such a choice because these fields can also be supported along a different holomorphic curve $\Sigma^{\prime}=D^{\prime} \cap D_{\text {vis }}$, where $D^{\prime}$ is some other divisor. Then, by choosing a trivial line bundle along each intersection curve $\Sigma_{i}=D \cap D_{i}$, which also includes $\Sigma$, the ample divisor will have no chiral intersections. This seemingly easy solution may be implemented if all the divisors are spin, i.e. when spinors exist globally on each divisor. However, such a scenario is definitely not generic because some divisors that commonly show up are non-spin, e.g. del Pezzo surfaces. Recall that if a divisor $D_{k}$ is non-spin, it will be forced to support a non-trivial line bundle in order to cancel the Freed-Witten anomaly [46]. In that case, as long as the corresponding intersection curve $\Sigma_{k}$ has self intersection equal $2\left(g\left(\Sigma_{k}\right)-1\right)$ inside the divisor $D_{k}$, the net chirality will still be zero but this is certainly more difficult to arrange.

Therefore, although the tension between chirality and Kahler moduli stabilization highlighted in [20] can in principle be resolved in the class of compactifications presented here, one would have to examine it on a case by case basis. In addition, even though some of these intersections may still support vector-like matter ${ }^{20}$ generically we expect that all vectorlike pairs have a large supersymmetric mass above the scale of gaugino condensation. In this case they can be integrated out, effectively yielding a non-perturbative superpotential that corresponds to a "pure glue" super Yang Mills gauge theory. ${ }^{21}$

\subsection{Mediation of supersymmetry breaking}

Since the moduli have been stabilized, one can also hope to make contact with more detailed aspects of particle physics and cosmology, at least in a broad sense. This requires

\footnotetext{
${ }^{20}$ When a holomorphic curve $\Sigma_{k}$ has genus zero and the line bundle along $\Sigma_{k}$ is trivial, there are no vector-like matter fields localized along $\Sigma_{k}$.

${ }^{21}$ In special cases there may exist extra symmetries that protect some vector-like pairs from receiving a high scale mass. In the companion paper [1] we will consider a scenario where the hidden sector gauge theory is a supersymmetric QCD with light vector-like pairs forming effective meson fields whose F-terms break supersymmetry spontaneously.
} 
a specification of the matter and gauge sector - both visible and hidden. Within the framework considered in the paper, we envision a four-dimensional matter and gauge sector arising from D3 branes at singularities or on stacks of D7-branes wrapping four-cycles in the Calabi-Yau threefold. From the analysis above, we have found that all moduli (including all Kähler moduli) can be stabilized by appropriate background fluxes as well as non-perturbative contributions arising from Euclidean D3-brane instantons or strong gauge dynamics on a stack of D7-branes, wrapping a single ample divisor. For the case of a visible sector arising from D7-branes, one could imagine stacks of intersecting D7-branes wrapping homologically different divisors, whose volumes are also fixed by the above mechanism, which may support the visible sector. The zero-mode spectrum of the visible sector can be made chiral if the Riemann surface that is at the locus of an intersection between two stacks of D7-branes has a non-trivial U(1) magnetic flux. Thus, it is possible to engineer (semi)-realistic matter and gauge spectra.

What can be said about the issues of supersymmetry breaking and its mediation to the visible sector? Within the framework above, supersymmetry is broken by anti D3branes at the tip of a warped throat generated in these flux compactifications, and could naturally be at the $\mathrm{TeV}$ scale. By gauge-gravity duality, this is dual to supersymmetry breaking states in an appropriate quiver gauge theory [43]. Depending on the location of the visible sector four-cycle relative to that of the anti D3-branes, different mediation mechanisms could dominate. For example, if the visible sector resides in the bulk of the Calabi-Yau then the mediation mechanism is moduli (gravity) mediation, suppressed by $1 / m_{\mathrm{Pl}}$ interactions $[31,32]$. Within such a setup, it has been argued that the warped throat between the visible and supersymmetry breaking sectors could give rise to sequestering [59]. It could happen that the visible sector also resides at the tip of the warped throat, in which case the dominant mediation mechanism is gauge mediation [26, 44] arising from messenger particles stretching between the visible and supersymmetry breaking sectors. One could also have more complicated setups in which the visible sector is comprised of a gauge sector on a stack of D7-branes which extends (partially) in the throat, and a chiral matter sector which resides in the bulk of the Calabi-Yau. In this case, the dominant mediation mechanism is gaugino mediation, from exchange of gauginos extending in the throat $[15,66]$. Thus, there can be a wealth of possibilities for low-energy particle physics. The situation in Type IIB compactifications is quite different from that in fluxless $G_{2}$ compactifications of $M$ theory where warping is not expected to be present. In $M$ theory compactifications to four dimensions, matter and gauge sectors live on three-cycles, and two three-cycles generically do not intersect in seven dimensions. Hence supersymmetry breaking in the hidden sector is mediated to the visible sector by moduli fields, giving rise to gravity mediation $[4,6]$.

\subsection{Dynamical solution to the strong CP-problem}

We now point out some consequences of the above moduli stabilization mechanism, which are different from the other mechanisms within Type IIB string theory, but have features similar to that in $M$ theory. As found in subsubsection 3.2.2, the Kähler modulus $\tau_{D}$ and 
its axion partner receive a mass of $\mathcal{O}(10) \times m_{3 / 2}$, while the remaining Kähler moduli receive masses of $\mathcal{O}\left(m_{3 / 2}\right)$.

The remaining axions turn out to be massless at this level, but will be eventually fixed by possible subdominant non-perturbative contributions to the superpotential as well as the Kähler potential. Note that even a single divisor can contribute an infinite number of terms to the superpotential when one takes into account multiple instanton wrappings, generating the following terms in the scalar potential

$$
m_{3 / 2} m_{p l}^{3} e^{K / 2} \sum_{j=1}^{\infty} C_{j} e^{-2 \pi j \sum m_{i} \tau_{i}} \cos \left[2 \pi \sum_{i}\left(\frac{n_{i}}{N}-j m_{i}\right) \chi_{i}\right],
$$

that will only ever slightly perturb the phase $\chi_{D}$ while giving exponentially suppressed masses to the individual axions $\chi_{i}$. Furthermore, possible non-BPS instanton contributions to the Kähler potential will generate additional but qualitatively similar non-perturbative terms in the scalar potential. Finally, for some axions the dominant effects that determine their masses may be generated by some low-scale strong gauge dynamics, similar to the QCD instanton effects in the visible sector discussed below. The important point is that complete axion stabilization does not require $h_{+}^{11}$ individual divisors to contribute to the superpotential. This is quite different from the standard scheme in which one has $h_{+}^{11}$ comparable non-perturbative terms in the superpotential, each depending on a different Kähler modulus. In that case, as stated earlier all axions, which are imaginary parts of the Kähler moduli, are stabilized at $\mathcal{O}\left(m_{3 / 2}\right)$.

This property of the axion spectrum has a crucially important consequence for a dynamical solution to the strong CP-problem. It has been a long-cherished dream in string phenomenology to use one of the numerous axions arising in a string compactification to be the QCD axion. One of the crucial requirements for the axion to be a QCD axion is that the dominant contribution to its potential must arise almost entirely from QCD instanton effects, with a mass given by $m_{a, \mathrm{QCD}} \sim \Lambda_{Q C D}^{2} / f_{a, \mathrm{QCD}} \ll m_{3 / 2}$. However, within the standard scheme mentioned above the axions are already stabilized at $\mathcal{O}\left(m_{3 / 2}\right)$, so none of them can serve as QCD axions. ${ }^{22}$ The moduli fixing mechanism described in this paper, on the other hand, only fixes one combination of axions at $\mathcal{O}(10) \times m_{3 / 2}$, so this serves as an excellent starting point for a solution to the strong CP-problem. The remaining axions are stabilized at exponentially suppressed scales relative to $m_{3 / 2}$, which is especially true when the subdominant non-perturbative contributions come from instantons as opposed to gaugino condensates, as should be evident from section 4 .

Once the subdominant non-perturbative terms are included, the masses of the remaining axion eigenstates will be given by

$$
m_{\Phi_{k}}^{2} \sim \mathcal{O}(1) \frac{m_{p l}^{3} m_{3 / 2}}{f_{k}^{2}} e^{K / 2} e^{-b_{k} \tau_{(k)}}
$$

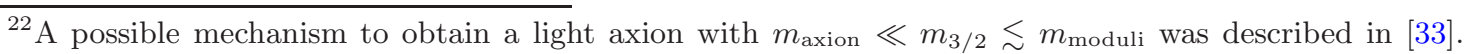
However, no explicit construction of a Calabi-Yau orientifold where such a scenario could be implemented was presented.
} 
where $\tau_{(k)}$ is the volume of the k-th divisor with a volume fixed at $\tau_{(k)}=\sum_{i} n_{i}^{(k)} \tau_{i}^{\min }$. In this framework $\theta_{Q C D}$ corresponds to a particular linear combination of the axions (the imaginary part of the QCD gauge kinetic function) plus a contribution from the phase of the quark mass matrix

$$
\theta_{Q C D}=2 \pi \sum_{i=1}^{h_{+}^{11}} n_{i}^{v i s} \chi_{i}+\arg \operatorname{det} m_{q}=\sum_{i=1}^{h_{+}^{11}} \frac{\Phi_{i}}{\tilde{f}_{i}}+\theta_{0},
$$

where $\theta_{0}=$ const is fixed by the vevs of the complex structure moduli and the dilaton. In the above, the integers $n_{i}^{v i s}$ specify the homology of the divisor supporting the visible sector SU(3) stack of D7 branes, or a GUT stack if one assumes grand unification, and $\tilde{f}_{i} \sim M_{G U T}$ are effective decay constants defined in [7]. Note that as long as the mass of lightest eigenstate inside the linear combination above satisfies

$$
m_{\Phi_{\text {lightest }}}^{2}<\frac{\Lambda_{Q C D}^{4}}{f_{a}^{2}} \times 10^{-10} \sim\left(10^{-14} e V\right)^{2}
$$

the QCD instanton effects will be strong enough to shift the vev $\Phi_{\text {lightest }}$ so that $\theta_{Q C D}<$ $10^{-10}$. This can be naturally satisfied when the mass of the lightest eigenstate is generated by a $D 3$ instanton that wraps a divisor of volume $\tau_{\text {inst }} \gtrsim \alpha_{G U T}^{-1} \approx 25$ for $m_{3 / 2} \approx 10 \mathrm{TeV}$, $f_{a} \approx M_{G U T}, V_{X} \approx 1000$. Thus, in this class of vacua, while all the geometric moduli are stabilized with masses $m_{\text {modili }}>O\left(m_{3 / 2}\right)$, the axions remain extremely light and provide a natural solution to the strong $\mathrm{CP}$ problem.

In the "standard" cosmological history, assuming a radiation-dominated phase after inflation, the axion relic density for $f_{a} \sim M_{G U T}$ is too high and typically overcloses the universe when the initial misalignment of the axion is $\mathcal{O}(1)$. However, when the cosmological history contains a moduli-dominated phase until just before BBN, as in the current scenario, the large entropy production from the late-time decaying moduli modifies the computation of the axion relic density and the bound on $f_{a}$ can be relaxed significantly $[7,45]$. Note, that for $f_{a} \sim M_{G U T}$ there is still $1-10 \%$ of tuning for the QCD axion's initial misalignment but it is not as severe as in the "standard" thermal scenario.

Furthermore, the presence of a multitude of very light axions, with masses distributed roughly linearly on a logarithmic scale, dynamically realizes the "String Axiverse" scenario discussed in [10], with many of their observable signatures. Note that the above features also hold for fluxless $G_{2}$ compactifications of $M$ theory since the moduli and axion fixing mechanism is very similar to that discussed in this paper. A full analysis of the solution to the strong CP-problem including observable consequences vis-a-vis the above mechanism has been carried out in [7].

\section{Conclusions}

In this work we have proposed an elegant and robust mechanism to stabilize all Kähler moduli in Type IIB string compactifications on Calabi-Yau orientifolds with D3/D7-branes, which is motivated from the analysis of moduli stabilization in fluxless $G_{2}$ compactifications 
of $M$ theory. This can be achieved with just one non-perturbative contribution to the superpotential, arising either from D3-instantons or from strong gauge dynamics on D7branes, wrapping an ample divisor with $\chi_{+}-\chi_{-}=1$. We have also argued that this scheme contains a simple resolution to the seeming tension between chirality and Kahler moduli stabilization and also naturally provides a dynamical solution to the strong CP-problem within string theory.

In order to break supersymmetry and obtain a de Sitter vacuum, we have followed the KKLT proposal and included explicit supersymmetry breaking terms in the scalar potential due to anti D3-branes. It has been argued that such a procedure may not be consistent from a microscopic point of view $[14,25]$. It is, therefore, important to study mechanisms in which supersymmetry is broken spontaneously and a de Sitter vacuum is obtained by adding additional $F$-term or $D$-term contributions to the potential. While this will not change the qualitative picture of moduli and axion stabilization and the resulting solution to the strong CP-problem outlined in the paper, it could have important effects for particle physics. This is being worked out in a companion paper [1].

\section{Acknowledgments}

We would like to thank Bobby Acharya, Ralph Blumenhagen, Ben Dundee, Joe Marsano, and Timo Weigand for useful discussions. K.B. and S.R. are supported by DOE grant DOE/ER/01545-885. P.K. is supported in part by the US Department of Energy under Contract DE-AC02-05CH11231 and in part by the US National Science Foundation Grant PHY-04-57315.

Open Access. This article is distributed under the terms of the Creative Commons Attribution Noncommercial License which permits any noncommercial use, distribution, and reproduction in any medium, provided the original author(s) and source are credited.

\section{References}

[1] K. Bobkov, V. Braun, P. Kumar, S. Raby, Part two, to appear.

[2] B.S. Acharya, A moduli fixing mechanism in M-theory, hep-th/0212294 [SPIRES].

[3] B.S. Acharya, F. Benini and R. Valandro, Fixing moduli in exact type IIA flux vacua, JHEP 02 (2007) 018 [hep-th/0607223] [SPIRES].

[4] B.S. Acharya and K. Bobkov, Kähler Independence of the G2-MSSM, JHEP 09 (2010) 001 [arXiv:0810.3285] [SPIRES].

[5] B.S. Acharya, K. Bobkov, G. Kane, P. Kumar and D. Vaman, An M-theory solution to the hierarchy problem, Phys. Rev. Lett. 97 (2006) 191601 [hep-th/0606262] [SPIRES].

[6] B.S. Acharya, K. Bobkov, G.L. Kane, P. Kumar and J. Shao, Explaining the electroweak scale and stabilizing moduli in M-theory, Phys. Rev. D 76 (2007) 126010 [hep-th/0701034] [SPIRES].

[7] B.S. Acharya, K. Bobkov and P. Kumar, An M-theory Solution to the Strong CP Problem and Constraints on the Axiverse, JHEP 11 (2010) 105 [arXiv:1004.5138] [SPIRES]. 
[8] A. Achucarro, B. de Carlos, J.A. Casas and L. Doplicher, de Sitter vacua from uplifting D-terms in effective supergravities from realistic strings, JHEP 06 (2006) 014 [hep-th/0601190] [SPIRES].

[9] S.P. de Alwis, Classical and Quantum SUSY Breaking Effects in IIB Local Models, JHEP 03 (2010) 078 [arXiv:0912.2950] [SPIRES].

[10] A. Arvanitaki, S. Dimopoulos, S. Dubovsky, N. Kaloper and J. March-Russell, String Axiverse, Phys. Rev. D 81 (2010) 123530 [arXiv:0905.4720] [SPIRES].

[11] V. Balasubramanian, P. Berglund, J.P. Conlon and F. Quevedo, Systematics of Moduli Stabilisation in Calabi-Yau Flux Compactifications, JHEP 03 (2005) 007 [hep-th/0502058] [SPIRES].

[12] A. Beauville, New trends in algebraic geometry (Warwick, 1996), London Math. Soc. Lecture Note Ser. 264, Cambridge University Press, Cambridge, U.K. (1999), pg. 13-17.

[13] M. Becker, G. Curio and A. Krause, de Sitter vacua from heterotic M-theory, Nucl. Phys. B 693 (2004) 223 [hep-th/0403027] [SPIRES].

[14] I. Bena, M. Graña and N. Halmagyi, On the Existence of Meta-stable Vacua in KlebanovStrassler, JHEP 09 (2010) 087 [arXiv:0912.3519] [SPIRES].

[15] F. Benini et al., Holographic Gauge Mediation, JHEP 12 (2009) 031 [arXiv:0903.0619] [SPIRES].

[16] M. Berg, M. Haack and B. Körs, On volume stabilization by quantum corrections, Phys. Rev. Lett. 96 (2006) 021601 [hep-th/0508171] [SPIRES].

[17] E. Bergshoeff, R. Kallosh, A.-K. Kashani-Poor, D. Sorokin and A. Tomasiello, An index for the Dirac operator on D3 branes with background fluxes, JHEP 10 (2005) 102 [hep-th/0507069] [SPIRES].

[18] R. Blumenhagen, Basics of F-theory from the Type IIB Perspective, Fortsch. Phys. 58 (2010) 820 [arXiv:1002.2836] [SPIRES].

[19] R. Blumenhagen, A. Collinucci and B. Jurke, On Instanton Effects in F-theory, JHEP 08 (2010) 079 [arXiv: 1002 .1894] [SPIRES].

[20] R. Blumenhagen, S. Moster and E. Plauschinn, Moduli Stabilisation versus Chirality for MSSM like Type IIB Orientifolds, JHEP 01 (2008) 058 [arXiv:0711.3389] [SPIRES].

[21] L. Borisov, Z. Hua, On Calabi-Yau threefolds with large nonabelian fundamental groups, Proc. Amer. Math. Soc. 136 (2008) 1549 [math. AG/0609728].

[22] R. Bousso and J. Polchinski, Quantization of four-form fluxes and dynamical neutralization of the cosmological constant, JHEP 06 (2000) 006 [hep-th/0004134] [SPIRES].

[23] V. Braun, On Free Quotients of Complete Intersection Calabi-Yau Manifolds, arXiv: 1003. 3235 [SPIRES].

[24] V. Braun, P. Candelas and R. Davies, A Three-Generation Calabi-Yau Manifold with Small Hodge Numbers, Fortsch. Phys. 58 (2010) 467 [arXiv:0910.5464] [SPIRES].

[25] R. Brustein and S.P. de Alwis, Moduli potentials in string compactifications with fluxes: Mapping the discretuum, Phys. Rev. D 69 (2004) 126006 [hep-th/0402088] [SPIRES].

[26] M. Buican, D. Malyshev and H. Verlinde, On the Geometry of Metastable Supersymmetry Breaking, JHEP 06 (2008) 108 [arXiv:0710.5519] [SPIRES]. 
[27] P.G. Camara, A. Font and L.E. Ibáñez, Fluxes, moduli fixing and MSSM-like vacua in a simple IIA orientifold, JHEP 09 (2005) 013 [hep-th/0506066] [SPIRES].

[28] P. Candelas and R. Davies, New Calabi-Yau Manifolds with Small Hodge Numbers, Fortsch. Phys. 58 (2010) 383 [arXiv:0809.4681] [SPIRES].

[29] B. de Carlos, S. Gurrieri, A. Lukas and A. Micu, Moduli stabilisation in heterotic string compactifications, JHEP 03 (2006) 005 [hep-th/0507173] [SPIRES].

[30] B. de Carlos, A. Lukas and S. Morris, Non-perturbative vacua for M-theory on $G_{2}$ manifolds, JHEP 12 (2004) 018 [hep-th/0409255] [SPIRES].

[31] K. Choi, A. Falkowski, H.P. Nilles and M. Olechowski, Soft supersymmetry breaking in KKLT flux compactification, Nucl. Phys. B 718 (2005) 113 [hep-th/0503216] [SPIRES].

[32] K. Choi, A. Falkowski, H.P. Nilles, M. Olechowski and S. Pokorski, Stability of flux compactifications and the pattern of supersymmetry breaking, JHEP 11 (2004) 076 [hep-th/0411066] [SPIRES].

[33] K. Choi and K.S. Jeong, String theoretic QCD axion with stabilized saxion and the pattern of supersymmetry breaking, JHEP 01 (2007) 103 [hep-th/0611279] [SPIRES].

[34] K. Choi, K.S. Jeong, S. Nakamura, K.-I. Okumura and M. Yamaguchi, Sparticle masses in deflected mirage mediation, JHEP 04 (2009) 107 [arXiv:0901.0052] [SPIRES].

[35] M. Cicoli, J.P. Conlon and F. Quevedo, General Analysis of LARGE Volume Scenarios with String Loop Moduli Stabilisation, JHEP 10 (2008) 105 [arXiv:0805.1029] [SPIRES].

[36] J.P. Conlon, F. Quevedo and K. Suruliz, Large-volume flux compactifications: Moduli spectrum and D3/D7 soft supersymmetry breaking, JHEP 08 (2005) 007 [hep-th/0505076] [SPIRES].

[37] G. Curio, A. Klemm, D. Lüst and S. Theisen, On the vacuum structure of type-II string compactifications on Calabi-Yau spaces with H-fluxes, Nucl. Phys. B 609 (2001) 3 [hep-th/0012213] [SPIRES].

[38] G. Curio, A. Krause and D. Lüst, Moduli stabilization in the heterotic / IIB discretuum, Fortsch. Phys. 54 (2006) 225 [hep-th/0502168] [SPIRES].

[39] K. Dasgupta, G. Rajesh and S. Sethi, M theory, orientifolds and G-flux, JHEP 08 (1999) 023 [hep-th/9908088] [SPIRES].

[40] F. Denef, M.R. Douglas and B. Florea, Building a better racetrack, JHEP 06 (2004) 034 [hep-th/0404257] [SPIRES].

[41] F. Denef, M.R. Douglas, B. Florea, A. Grassi and S. Kachru, Fixing all moduli in a simple F-theory compactification, Adv. Theor. Math. Phys. 9 (2005) 861 [hep-th/0503124] [SPIRES].

[42] O. DeWolfe, A. Giryavets, S. Kachru and W. Taylor, Type IIA moduli stabilization, JHEP 07 (2005) 066 [hep-th/0505160] [SPIRES].

[43] O. DeWolfe, S. Kachru and M. Mulligan, A Gravity Dual of Metastable Dynamical Supersymmetry Breaking, Phys. Rev. D 77 (2008) 065011 [arXiv:0801.1520] [SPIRES].

[44] D.-E. Diaconescu, B. Florea, S. Kachru and P. Svrček, Gauge - mediated supersymmetry breaking in string compactifications, JHEP 02 (2006) 020 [hep-th/0512170] [SPIRES].

[45] P. Fox, A. Pierce and S.D. Thomas, Probing a QCD string axion with precision cosmological measurements, hep-th/0409059 [SPIRES]. 
[46] D.S. Freed and E. Witten, Anomalies in string theory with D-branes, hep-th/9907189 [SPIRES].

[47] D. Gallego and M. Serone, An Effective Description of the Landscape - I, JHEP 01 (2009) 056 [arXiv:0812.0369] [SPIRES].

[48] D. Gallego and M. Serone, An Effective Description of the Landscape - II, JHEP 06 (2009) 057 [arXiv: 0904.2537] [SPIRES].

[49] G. von Gersdorff and A. Hebecker, Kähler corrections for the volume modulus of flux compactifications, Phys. Lett. B 624 (2005) 270 [hep-th/0507131] [SPIRES].

[50] S.B. Giddings, S. Kachru and J. Polchinski, Hierarchies from fluxes in string compactifications, Phys. Rev. D 66 (2002) 106006 [hep-th/0105097] [SPIRES].

[51] A. Giryavets, S. Kachru and P.K. Tripathy, On the taxonomy of flux vacua, JHEP 08 (2004) 002 [hep-th/0404243] [SPIRES].

[52] A. Giryavets, S. Kachru, P.K. Tripathy and S.P. Trivedi, Flux compactifications on Calabi-Yau threefolds, JHEP 04 (2004) 003 [hep-th/0312104] [SPIRES].

[53] L. Görlich, S. Kachru, P.K. Tripathy and S.P. Trivedi, Gaugino condensation and nonperturbative superpotentials in flux compactifications, JHEP 12 (2004) 074 [hep-th/0407130] [SPIRES].

[54] G.M. Grauel, G. Pfister, H. Schönemann, Singular 3.0, Centre for Computer Algebra, University of Kaiserslauten, Kaiserslauten, Germany (2005), http://www.singular.uni-kl.de.

[55] S. Gukov, S. Kachru, X. Liu and L. McAllister, Heterotic moduli stabilization with fractional Chern-Simons invariants, Phys. Rev. D 69 (2004) 086008 [hep-th/0310159] [SPIRES].

[56] S. Gukov, C. Vafa and E. Witten, CFT's from Calabi-Yau four-folds, Nucl. Phys. B 584 (2000) 69 [hep-th/9906070] [SPIRES].

[57] Z. Hua, Classification of free actions on complete intersections of four quadrics [arXiv: 0707.4339].

[58] S. Kachru, R. Kallosh, A.D. Linde and S.P. Trivedi, de Sitter vacua in string theory, Phys. Rev. D 68 (2003) 046005 [hep-th/0301240] [SPIRES].

[59] S. Kachru, L. McAllister and R. Sundrum, Sequestering in string theory, JHEP 10 (2007) 013 [hep-th/0703105] [SPIRES].

[60] S. Kachru, M.B. Schulz and S. Trivedi, Moduli stabilization from fluxes in a simple IIB orientifold, JHEP 10 (2003) 007 [hep-th/0201028] [SPIRES].

[61] I.R. Klebanov and M.J. Strassler, Supergravity and a confining gauge theory: Duality cascades and $\chi S B$-resolution of naked singularities, JHEP 08 (2000) 052 [hep-th/0007191] [SPIRES].

[62] O. Lebedev, H.P. Nilles and M. Ratz, de Sitter vacua from matter superpotentials, Phys. Lett. B 636 (2006) 126 [hep-th/0603047] [SPIRES].

[63] M.M. Lopes, R. Pardini, Numerical Campedelli surfaces with fundamental group of order 9, J. Eur. Math. Soc. 10 (2008) 457.

[64] D. Lüst, S. Reffert, E. Scheidegger, W. Schulgin and S. Stieberger, Moduli stabilization in type IIB orientifolds. II, Nucl. Phys. B 766 (2007) 178 [hep-th/0609013] [SPIRES].

[65] D. Lüst, S. Reffert, W. Schulgin and S. Stieberger, Moduli stabilization in type IIB orientifolds. I: Orbifold limits, Nucl. Phys. B 766 (2007) 68 [hep-th/0506090] [SPIRES]. 
[66] P. McGuirk, G. Shiu and Y. Sumitomo, Holographic gauge mediation via strongly coupled messengers, Phys. Rev. D 81 (2010) 026005 [arXiv:0911.0019] [SPIRES].

[67] A. Micu, Moduli Stabilisation in Heterotic Models with Standard Embedding, JHEP 01 (2010) 011 [arXiv:0911.2311] [SPIRES].

[68] J. Park, D3 instantons in Calabi-Yau orientifolds with(out) fluxes, Eur. Phys. J. C 67 (2010) 263 [hep-th/0507091] [SPIRES].

[69] D. Robbins and S. Sethi, A barren landscape, Phys. Rev. D 71 (2005) 046008 [hep-th/0405011] [SPIRES].

[70] E. Silverstein, Simple de Sitter Solutions, Phys. Rev. D 77 (2008) 106006 [arXiv:0712.1196] [SPIRES].

[71] T.R. Taylor and C. Vafa, RR flux on Calabi-Yau and partial supersymmetry breaking, Phys. Lett. B 474 (2000) 130 [hep-th/9912152] [SPIRES].

[72] G. Villadoro and F. Zwirner, $N=1$ effective potential from dual type-IIA D6/O6 orientifolds with general fluxes, JHEP 06 (2005) 047 [hep-th/0503169] [SPIRES].

[73] E. Witten, Non-Perturbative Superpotentials In String Theory, Nucl. Phys. B 474 (1996) 343 [hep-th/9604030] [SPIRES]. 